\title{
Generalized Eigenfunctions for Dirac Operators Near Criticality
}

\author{
Peter Pickl*
}

October 14, 2018

\begin{abstract}
Critical Dirac operators are those which have eigenfunctions and/or resonances for $E=m$. We estimate the behavior of the generalized eigenfunctions of critical Dirac operators under small perturbations of the potential. The estimates are done in the $L^{\infty}$-norm. We show that for small $k$ the generalized eigenfunctions are in leading order multiples of the respective eigenfunctions and/or resonances. We also estimate the $k$-derivatives which are important for estimating decay. The method also applies for other differential operators (for example Schrödinger operators).
\end{abstract}

${ }^{*}$ Institut für theoretische Physik, Universität Wien, Boltzmanngasse 5, 1090 Vienna, Austria E-mail: pickl@mathematik.uni-muenchen.de 


\section{Contents}

1 Introduction 3

2 Solutions of the Lippmann Schwinger Equation

2.1 Critical Potentials . . . . . . . . . . . . . . . . . . . 6 6

3 Generalized Eigenfunctions for Critical Potentials with Small Perturbations 7

4 Discussion of the Result 11

5 Proof of the Theorem 12

$6 \quad k$-Derivatives 20 


\section{Introduction}

Expansion into generalized eigenfunctions of Schrödinger- or Dirac operators is an important technique in physics to get control on the time evolution of wave functions. Moreover it was used to establish completeness in scattering theory (see for example [8]) as well as to establish the so called Flux Across Surfaces Theorem which lies at the basis of scattering theory (2, 25, 3, 5, 4]). Of particular interest is however the case which in scattering theory is normally excluded, namely when resonances occur and/or there is an eigenvalue on the edge of the continuous spectrum. We say then that the operator is critical. Such a critical situation occurs very naturally for the Dirac operator with a very strong time dependent external potential which is compactly supported. In this case the famous relativistic effect of pair creation can happen. This is best be pictured by considering a time dependent eigenvalue of the time dependent Dirac operator. For not so strong fields eigenvalues may lie within the spectral gap $\left(-m c^{2}, m c^{2}\right)$. When the potential adiabatically increases (decreases) an eigenvalue increases (decreases) and eventually touches the positive (negative) edge, i.e. $\pm m c^{2}(\hat{=} k=0)$ of the continuous spectrum. On the edge the eigenvalue becomes either a resonance or stays an eigenvalue. Generically on the upper edge the eigenvalue stays an eigenvalue, while on the lower edge it typically ceases to be an eigenvalue ([11] and [23] for the Schrödinger case). When the potential increases further, the eigenvalue disappears.

The question then is what happens to the critical bound state, i.e. the state corresponding to the eigenvalue on the edge. Does it scatter? If so pair creation is achieved. This situation has been extensively studied in the physics literature 1, 19, 6, 7, 15. It has also been studied in the mathematical physics literature [12, 13, 18 but the mathematical proof of pair creation was still lacking until recently. In 16 we provide a proof of the effect of pair creation in an adiabatically changing potential where the scattering behavior of the critical bound state is controlled by generalized eigenfunctions using the results of the present paper in an essential way. This then is the physical setting of the problem studied here. What one needs and what we provide here is the control of the behavior of the generalized eigenfunctions around and at criticality. The reader should have in mind the stationary phase argument to understand what kind of control one needs to control the time evolution of wave functions. One needs upper bounds on the $L^{\infty}$-norm on the $k$-derivatives of the generalized eigenfunctions. The quality of that bound is essential for the quality of the bound on the "speed of decay" of the wave function, see e.g. (7) below.

We emphasize that the relevance of this question is by no means restricted to the Dirac operator case which we just discussed. While our method can be applied as well to other operators we formulate our results and proof for the Dirac case because we have the particular application of pair creation in mind. Moreover we shall use the Green's function of the free operator in some essential way, which is more complicated in the Dirac case than in the Schrödinger case: The Green's function of the Dirac Operator is a matrix-multiple of the Green's function of the Laplacian plus some extra terms. That is the Schrödinger case can be handled following the Dirac case by essentially omission of some extra terms.

Previous works deal exclusively with the resolvent $[9$ or directly with eigenfunctions 22 at criticality, but nothing is known about the behavior in the neighborhood of criticality, which is generically the relevant question.

From these results it is clear that the normalized (which means normalized to delta functions in this case) generalized eigenfunctions of a critical potential diverge as $k$ goes to zero. We need to generalize this to a family of operators the members of which vary in the neighborhood of a critical potential. In fact one should think of the family as arising from the perturbations of a critical potential. We need to control the behavior of the generalized eigenfunctions in dependence of $k$ and the perturbation $B$ of the critical potential. To be clear the generalized eigenfunctions depend on $k$ and $B$.

The main result of this paper is Theorem 3.4. where we give an estimate of the $L^{\infty}$-norm of the normalized generalized eigenfunctions in dependence of $k$ and $B$ when the critical potential has a bound state at the edge.

The behavior is different from the case when the critical potential has no bound state at the edge. The latter situation is also dealt with in this paper. The result is spelled out in Theorem 3.5 .

Recently in [10] a question similar to ours has been asked, namely to estimate the decay of a critical bound state. While our method is different, it is more general then [10] and gives, concerning the decay, the same result [14, [15, 16.

We shall now be more detailed. We shall use units where $c=m=\hbar=1$. Furthermore throughout 
the paper the letters $C$ and $C_{n}, n \in \mathbb{N}_{0}$ will be used for various constants that need not be identical even within the same equation. Finally the absolute value of any vector $\mathbf{x} \in \mathbb{R}^{3}$ shall be denoted by $x$. by

The one particle Dirac operator $D$ with external potential in the "standard representation" is defined

$$
D \psi=-i \sum_{l=1}^{3} \alpha_{l} \partial_{l} \psi+A \psi+\beta \psi \equiv\left(D^{0}+A\right) \psi,
$$

where the $4 \times 4$-matrices $\alpha_{l}$ and $\beta$ are defined via

$$
\alpha_{l}=\left(\begin{array}{cc}
0 & \sigma_{l} \\
\sigma_{l} & 0
\end{array}\right) ; \beta=\left(\begin{array}{cc}
1 & 0 \\
0 & -1
\end{array}\right) ; l=1,2,3
$$

with $\sigma_{l}$ being the Pauli matrices

$$
\sigma_{1}=\left(\begin{array}{cc}
1 & 0 \\
0 & -1
\end{array}\right) ; \sigma_{2}=\left(\begin{array}{cc}
0 & 1 \\
1 & 0
\end{array}\right) ; \sigma_{3}=\left(\begin{array}{cc}
0 & -i \\
i & 0
\end{array}\right)
$$

and

$$
A=A_{0}+\sum_{l=1}^{3} \alpha_{l} A_{l}
$$

for the four potential $A_{\mu}$ ( $A$ is usually denoted by $A$ in the literature).

Note that $\psi$ is a 4 -vector valued function and the underlying Hilbert space is $\mathcal{H}=L^{2}\left(\mathbb{R}^{3}\right)^{4}$.

We are interested in the (generalized) eigenfunctions of the Dirac operator, i.e. $L^{\infty}$-solutions of

$$
E \phi_{E}=D \phi_{E}
$$

for $E \in \mathbb{R}$.

One can show (see Lemma 2.2 below), that for a rather general class of potentials $A$ any such solution solves the so called Lippmann Schwinger equation

$$
\phi_{E}(\mathbf{x})=\chi_{E}(\mathbf{x})+\int G_{E}^{+}(\mathbf{x}-\mathbf{y}) A(\mathbf{y}) \phi_{E}(\mathbf{y}) d^{3} y
$$

where $G_{E}^{+}$are the kernels of $\left(E-D^{0}\right)^{-1}=\lim _{\delta \rightarrow 0}\left(E-D^{0}+i \delta\right)^{-1}$ and the $\chi_{E} \in L^{\infty}$ are solutions of

$$
E \chi_{E}=D^{0} \chi_{E}
$$

Let us heuristically explain the main point of this paper. We are interested in the behavior of the $L^{\infty}$-norm of the $L^{\infty}$-solutions of (5) with energy $E_{k}= \pm \sqrt{k^{2}+1}$ for critical potential $A$ plus some small perturbation $B$. The $L^{\infty}$-solutions of (6) for $E_{k}= \pm \sqrt{k^{2}+1}$ are $e^{i \mathbf{k} \cdot \mathbf{x}}$ multiplied with some (kdependent) spinor. For any $\mathbf{k} \in \mathbb{R}^{3}$ and any $\operatorname{sign}$ of $E$ there exist two different $L^{\infty}$-normalized $\chi(j, \mathbf{k}, \cdot)$ (spin degeneration, see [26]). To distinguish between these different solutions we have introduced the spin index $j$ which is 1 or 2 for positive energies and 3 or 4 for negative energies.

It is already known (see [5]) that for any $B$ and any $\chi(j, \mathbf{k}, \cdot)$ (so for any $\left.(\mathbf{k}, j) \in \mathbb{R}^{3} \times\{1,2,3,4\}\right)$ there exists (up to linearity) exactly one solution $\phi(A+B, j, \mathbf{k}, \cdot)$ of (5). We have (see again [5]) for non-critical $A+B$ that

but for $B \equiv 0($ see $[9])$

$$
\sup _{(\mathbf{k}, j) \in \mathbb{R}^{3} \times\{1,2,3,4\}}\|\phi(A+B, j, \mathbf{k}, \cdot)\|_{\infty}<\infty,
$$

$$
\lim _{k \rightarrow 0} \sup _{j=1,2,3,4}\|\phi(A, j, \mathbf{k}, \cdot)\|_{\infty}=\infty .
$$

The central part of this paper is to generalize this result and estimate the $B$ and $k$ behavior of $\| \phi(A+$ $B, j, \mathbf{k}, \cdot) \|_{\infty}$ for $(B, \mathbf{k})$ around $(0,0)$. We will show, that in the generic case, which means that the Dirac operator with potential $A$ has a bound state $\Phi \in L^{2}$ with energy 1 or -1 (for simplicity we give the formula in the case that the bound state is non-degenerate)

$$
\|\phi(A+B, j, \mathbf{k}, \cdot)\|_{\infty} \leq \frac{C k}{\left|\langle\Phi, B, \Phi\rangle-C_{2} k^{2}\right|+k^{3}}
$$


for some real constants $C, C_{2}, C_{3}$ uniform in $\mathbf{k}$ and $B$ (c.f. Corollary 3.7 ).

This result is an important step forward in controlling the propagation of wave functions under the influence of critical potentials with small perturbations via eigenfunction expansion. One application of this is the decay of the QED vacuum via spontaneous (=adiabatic) pair creation under the influence of an adiabatic external potential. Adiabatic pair creation occurs just when the external potential becomes overcritical, so (7) is useful to estimate the rate and the momentum spectrum of the pairs.

The paper is organized as follows. In the following section 3 we give the Lippmann Schwinger equation adapted to our setting and give some relevant formulas. In section 2 we formulate the main results as well as a Corollary which is formulated for a somewhat easier situation and which has to grasp the meaning of the main result. In section 4 we discuss the physical meaning of the Corollary. Section 5 gives the proof, in section 6 we generalize our result to the $k$-derivatives of the generalized eigenfunctions which are needed to achieve good control on the time evolution of wave functions (stationary phase). Some technical details have been put into the appendix.

\section{Solutions of the Lippmann Schwinger Equation}

In view of (5) we define

Definition 2.1 Let $\mathcal{B} \subset L^{\infty}$ be the Banach space of functions tending uniformly to zero for $x \rightarrow \infty$. Let for $A \in L^{1} \cap L^{\infty}$ and $E \in \mathbb{R}$ the $T_{E}^{A}: L^{\infty} \rightarrow \mathcal{B}$ be the operator defined by

$$
\begin{aligned}
T_{E}^{A} f(\mathbf{x}) & :=\int G_{E}^{+}(\mathbf{y}) A(\mathbf{x}-\mathbf{y}) f(\mathbf{x}-\mathbf{y}) d^{3} y \\
& =-\int G_{E}^{+}(\mathbf{x}-\mathbf{y}) A(\mathbf{y}) f(\mathbf{y}) d^{3} y
\end{aligned}
$$

By this definition (5) can be written as

$$
\left(1-T_{E}^{A}\right) \phi_{E}(\mathbf{x})=\chi_{E}(\mathbf{x})
$$

furthermore

$$
T_{E}^{A+B}=T_{E}^{A}+T_{E}^{B}
$$

Note that for $|E|<1$ there exists only the trivial solution of the free Dirac eigenvalue equation (6), hence for $|E|<1$ (9) reads

$$
\left(1-T_{E}^{A}\right) \phi_{E}=0 .
$$

The proof that $T_{E}^{A}$ maps $L^{\infty}$ into $\mathcal{B}$ can be found in [5].

Lemma 2.2 Let $A \in L^{\infty} \cap L^{1}$ be Hölder continuous of degree one. Then

(a) Any $\phi_{E} \in L^{\infty}$ satisfies (4) if and only if $\left(1-T_{E}^{A}\right) \phi_{E}$ satisfies (6),

(b) for any solution $\phi_{E} \in \mathcal{B}$ of (9) we have that $|E| \leq 1$ and thus $\Phi_{E}$ satisfies (11).

(c) for any nontrivial solution $\phi_{E} \in L^{\infty} \backslash \mathcal{B}$ of (9) we have that $|E| \geq 1$ and $\Phi_{E}$ satisfies (9) with nontrivial $\chi_{E}$.

Proof: The statement (a) is well know. Since the proof is short and easy we shall give it now. Let $A \in L^{\infty} \cap L^{1}$ be Hölder continuous of degree one. Note that any $L^{\infty}$ solution of (4) is continuous (even partially differentiable, since $D^{0} f \in L^{\infty}$ ). Furthermore $T_{E}^{A} f$ is continuous for any $f \in L^{\infty}$ (see for example [5]), hence any solution of (9) is continuous.

By definition of $T_{E}^{A}$ we have for any continuous $f \in L^{\infty}$ that

$$
\left(E-D^{0}\right) T_{E}^{A} f=\int \delta(\mathbf{x}-\mathbf{y}) A(\mathbf{y}) f(\mathbf{y}) d^{3} y=A f,
$$


hence

$$
\left(D^{0}-E\right)\left(1-T_{E}^{A}\right) f=\left(D^{0}-E+A\right) f=(D-E) f .
$$

It follows, that $(D-E) f=0 \Leftrightarrow\left(D^{0}-E\right)\left(1-T_{E}^{A}\right) f=0$. In other words, $f$ is solution of (41) if and only if $\left(1-T_{E}^{A}\right) f$ is solution of (6), i.e. $f$ solves (9) with some $\chi_{E}$ solving (6).

The proof of (b) is as follows: Let $\phi_{E} \in \mathcal{B}$ be solution of (15). Since $T_{E}^{A}$ maps $L^{\infty}$ into $\mathcal{B}$ it follows that $\chi_{E}(\mathbf{x}) \in \mathcal{B}$. Since there exist no solutions $\chi_{E}(\mathbf{x}) \in \mathcal{B}$ of (6) but the trivial one, it follows that $\chi_{E}(\mathbf{x}) \equiv 0$. With (5) we get (11). Due to [26] no solutions of (11) exist for the potentials we consider for $|E|>1$ and (b) follows.

For (c) recall that $T_{E}^{A}$ maps $L^{\infty} \rightarrow$

mathcalB, hence $\phi_{E} \in L^{\infty} \backslash \mathcal{B}$ implies that $\chi_{E} \in L^{\infty} \backslash \mathcal{B}$, in particular $\chi_{E}$ is nontrivial. Nontrivial solutions of (6) exist only for $|E| \geq 1$ and (c) follows.

\subsection{Critical Potentials}

In view of Lemma 2.2 we have that for $|E|>1$ there only exist solutions of (4) which also solve (9) with nontrivial $\chi_{E}$. For $|E|<1$ there only exist solutions of (4) which also solve (11). For $E= \pm 1$ - depending on the potential $A$ - both kinds of eigenfunctions may exist. It is known, that generically solutions of (4) with $E= \pm 1$ also solve (9) with nontrivial $\chi_{ \pm 1}$.

Potentials where the $E= \pm 1$ solutions of (4) also solve (11) are called critical in the literature. We will focus on positive energy only, so "critical" means here, that there is a $E=+1$ solution of (4) which solves (11). All results can be obtained equivalently for negative energies, too (see Remark 3.6).

Definition 2.3 We call a 4-potential A critical if and only if there exist solutions $\Phi$ of (2.2) with energy $E=1$ (i.e. $\left.\left(1-T_{1}^{A}\right) \Phi=0\right)$. We denote the set of these solutions by $\mathcal{N}$

$$
\mathcal{N}:=\left\{\Phi \in \mathcal{B}:\left(1-T_{1}^{A}\right) \Phi=0\right\}
$$

The elements of $\mathcal{N}$ can be bound states (i.e. $L^{2}$-solutions of (11)) or so called resonances (i.e. not square integrable $\mathcal{B}$-solutions of (11)). Next we shall find a formula which distinguishes between these two different cases and which shall play a crucial role later on.

Let $\Phi \in \mathcal{N}$, i.e.

$$
\Phi(\mathbf{x})=\int G_{1}^{+}(\mathbf{y}) A(\mathbf{x}-\mathbf{y}) \Phi(\mathbf{x}-\mathbf{y}) d^{3} y
$$

The explicit form of $G_{E}^{+}$can be found in 26

$$
G_{E}^{+}(\mathbf{x})=\frac{1}{4 \pi} e^{i k x}\left(-x^{-1}\left(E_{k}+\sum_{j=1}^{3} \alpha_{j} k \frac{x_{j}}{x}+\beta\right)-i x^{-2} \sum_{j=1}^{3} \alpha_{j} \frac{x_{j}}{x}\right)
$$

where $k=\sqrt{E^{2}-1}$ (hence $E=1$ implies $k=0$ ). Thus

$$
\begin{aligned}
\Phi(\mathbf{x})= & -\int \frac{1}{4 \pi} y^{-1}\left(1+\beta+i \sum_{j=1}^{3} \alpha_{j} \frac{y_{j}}{y^{2}}\right) A(\mathbf{x}-\mathbf{y}) \Phi(\mathbf{x}-\mathbf{y}) d^{3} y \\
= & \left.-\int \frac{1}{4 \pi}\left(\left(y^{-1}-x^{-1}\right)(1+\beta)+i \sum_{j=1}^{3} \alpha_{j} \frac{y_{j}}{y^{3}}\right)\right) A(\mathbf{x}-\mathbf{y}) \Phi(\mathbf{x}-\mathbf{y}) d^{3} y \\
& -x^{-1} \int \frac{1}{4 \pi}(1+\beta) A(\mathbf{x}-\mathbf{y}) \Phi(\mathbf{x}-\mathbf{y}) d^{3} y \\
=: & \Phi_{1}(\mathbf{x})+\Phi_{2}(\mathbf{x}) .
\end{aligned}
$$

One can show that for large $x$ the $\Phi_{1}$ decays at least as fast as $x^{-2}$. The heuristics for that is rather clear. $A$ decays fast, thus for large $x$ the integrand is negligible if $|\mathbf{y}-\mathbf{x}| \gg 1$. Thus the factor 
$y^{-1}-x^{-1}=(x-y) /(x y)$ is for large $x$ of order $x^{-2}$, so $\Phi_{1}(\mathbf{x})$ decays at least as fast as $x^{-2}$. A rigorous proof for that is given in the appendix.

To find out, whether $\Phi \in L^{2}$ it is left to control $\Phi_{2}(\mathbf{x})$. The decay of $\Phi_{2}(\mathbf{x})$ depends on the spinor components of $\Phi(\mathbf{y})$. Setting

$$
\lambda(\Phi):=\int(1+\beta) A(\mathbf{y}) \Phi(\mathbf{y}) d^{3} y
$$

there are two alternatives: Either the spinor components of $\Phi(\mathbf{y})$ are such that $\lambda(\Phi) \neq 0$ and thus $\Phi_{2}(\mathbf{x})$ is of order $x^{-1}$ and thus $\Phi \notin L^{2}$ or such that $\lambda(\Phi)=0$ and thus $\Phi \in L^{2}$. The final result of this paper will depend on whether $\lambda(\Phi)$ is equal to zero or not, i.e. if $\Phi \in L^{2}$ or not.

This dichotomy can be compared to the results of [11, where the behavior of bound states of an almost critical potential is studied. This behavior crucially depends on the fact if $\lambda=0$ or not. Further explanation how this is related to our results shall be given below.

Notation 2.4 Below we will restrict ourselves to potentials where either $\lambda(\Phi)=0$ for all $\Phi \in \mathcal{N}$, or $\lambda(\Phi) \neq 0$ for all $\Phi \in \mathcal{N}$. To distinguish between these two cases we define

$$
\bar{\lambda}:= \begin{cases}0, & \text { if } \lambda(\Phi)=0 \text { for all } \Phi \in \mathcal{N} \\ 1, & \text { is } \lambda(\Phi) \neq 0 \text { for all } \Phi \in \mathcal{N}\end{cases}
$$

This restriction rules out potentials with $\operatorname{dim} \mathcal{N}>2$ and $\lambda(\Phi) \neq 0$ : If $\operatorname{dim} \mathcal{N}>2$ one can always find a $\Phi \in \mathcal{N}$ such that $\lambda(\Phi)=0$ using linearity of (15) and the fact that the kernel of $1+\beta$ is two dimensional (hence the image of $1+\beta$ is two dimensional so $\lambda(\Phi)$ is always an element of a two dimensional subspace of $\left.\mathbb{C}^{4}\right)$.

\section{Generalized Eigenfunctions for Critical Potentials with Small Perturbations}

Definition 3.1 For any selfadjoint matrix valued multiplication operator $A \in L^{1}$ let the (pseudo) scalar product $\langle\cdot, A, \cdot\rangle: L^{\infty} \times L^{\infty} \rightarrow \mathbb{C}$ be given by

$$
\langle f, A, g\rangle:=\int f^{\dagger}(\mathbf{x}) A(\mathbf{x}) g(\mathbf{x}) d^{3} x
$$

For any $K>0$ let the set $\mathcal{W}_{K} \subset L^{\infty} \cap L^{1}$ be given by

$$
B \in \mathcal{W}_{K} \Leftrightarrow B \in L^{\infty} \cap L^{1} \text { with } \frac{\|\Phi\|_{\infty}^{2}\left(\|B\|_{1}+\|B\|_{\infty}\right)^{2}}{|\langle\Phi, B, \Phi\rangle|} \leq K \text { for all } \Phi \in \mathcal{N} \text {. }
$$

For any critical $A \in L^{1} \cap L^{\infty}$ we define the following subspaces of $\mathcal{B}$

$$
\begin{aligned}
\mathcal{M}^{\|} & :=A \mathcal{N}:=\{A \Phi: \Phi \in \mathcal{N}\} \subset L^{2} \\
\mathcal{M}^{\perp} & :=\left\{m^{\perp} \in \mathcal{B}:\left\langle m^{\perp}, A, \Phi\right\rangle=0 \forall \Phi \in \mathcal{N}\right\} .
\end{aligned}
$$

In the following we will restrict our observations to critical potentials which satisfy some additional (weak) conditions.

Definition 3.2 Let $\mathcal{C}$ be the set of critical potentials defined by $A \in \mathcal{C}$ if and only if

(a) A is critical and Hölder continuous of degree one,

(b) $(1+|\cdot|)^{2} A \in L^{1} \cap L^{\infty}$,

(c) $\mathcal{N} \cap \mathcal{M}^{\perp}=\{0\}$,

(d) either $\mathcal{N} \subset L^{2}$ or $\mathcal{N} \cap L^{2}=\{0\}$, 
(e) either

$$
\int A(\mathbf{x}) \Phi(\mathbf{x}) d^{3} x \neq 0
$$

or

$$
(1-i \beta) \int A(\mathbf{x}) \Phi(\mathbf{x}) \mathbf{x} d^{3} x \neq 0 .
$$

Remark 3.3 It is rather clear that either (16) or (17) are satisfied for almost every critical potential. For example if $\Phi$ is a ground state and $A$ is purely electric (=multiple of the unit matrix) and positive, the Perron-Frobenius Theorem implies that (16) holds.

Furthermore we have for any purely electric, positive critical, "short range" potential (which means in our case $\left.(1+|\cdot|)^{2} A \in L^{1} \cap L^{\infty}\right)$ that $\mathcal{N} \cap \mathcal{M}^{\perp}=\{0\}$ : Obviously $\langle\Phi, A, \Phi\rangle>0$ for any positive electric potential $A$ and any $\Phi \in \mathcal{N}$. Small perturbations do not significantly change $\langle\Phi, A, \Phi\rangle$. Hence the set of critical, "short range" potentials with $\mathcal{N} \cap \mathcal{M}^{\perp}=\{0\}$ is not small. It seems that almost every critical "short range" (in the given sense) potential lies in $\mathcal{C}$.

In this paper we wish to estimate the generalized eigenfunctions of the Dirac operator with potentials $A+B$ where $A \in \mathcal{C}$ and $B \in \mathcal{W}_{K}$ for some (small) $K$. The generalized eigenfunctions are the respective solutions of (9), i.e. solutions of

$$
\left(1-T_{ \pm E_{k}}^{A+B}\right) \phi(A+B, j, \mathbf{k}, \cdot)=\chi(j, \mathbf{k}, \cdot),
$$

where the $\chi(j, \mathbf{k}, \cdot)$ are the $L^{\infty}$-normalized generalized eigenfunctions of the free Dirac operator with momentum $\mathbf{k}$ and spin $j, E_{k}=\sqrt{k^{2}+1}$ and the sign + holds for $j=1,2$, the sign - holds for $j=3,4$. For "small" $B$ we have - similar as in the $B=0$-case (see 9]) that the generalized eigenfunctions are of leading order a multiple of some element of $\mathcal{N}$. Which element may depend on $B, \mathbf{k}$ and $j$. We will estimate the divergent behavior of this element in dependence of $B, \mathbf{k}$ and $j$ and the $L^{\infty}$-norm of the generalized eigenfunctions minus their leading order $\mathcal{N}$-part. As mentioned above, that behavior of the generalized eigenfunctions depends crucially on the fact is $\bar{\lambda}=0$ or $\bar{\lambda}=1$. It is convenient to give two Theorems separating these two different cases. For $\bar{\lambda}=0$ we have

Theorem 3.4 Let $A \in \mathcal{C}$ with $\bar{\lambda}=0$ (i.e. $\mathcal{N} \subset L^{2}$ ). Then there exist constants $C, K, k_{0}>0$ and a selfadjoint linear map $\widehat{R}: \mathcal{N} \rightarrow \mathcal{N}$ such that for any $\mathbf{k} \in \mathbb{R}^{3}$ with $k<k_{0}, j=1,2$, any potential $B \in \mathcal{W}_{K}$ there exists a $\Phi_{j, \mathbf{k}}^{B} \in \mathcal{N}$ with

$$
\left\|\Phi_{j, \mathbf{k}}^{B}\right\| \leq C\left(k+\|B\|_{1}\right)\left(\inf _{\Phi \in \mathcal{N},\|\Phi\|=1}\left\|\left(P_{\mathcal{N}}^{\|} B+\widehat{R} k^{2}\right) \Phi\right\|+k^{3}\right)^{-1}
$$

and (c.f. (18))

$$
\left\|\phi(A+B, j, \mathbf{k}, \cdot)-\Phi_{j, \mathbf{k}}^{B}\right\|_{\infty}<C+C k\left(\|B\|_{1}+\|B\|_{\infty}\right)\left(\inf _{\Phi \in \mathcal{N},\|\Phi\|=1}\left\|\left(P_{\mathcal{N}}^{\|} B+\widehat{R} k^{2}\right) \Phi\right\|+k^{3}\right)^{-1} .
$$

For $\bar{\lambda}=1$ we have

Theorem 3.5 Let $A \in \mathcal{C}$ and $\bar{\lambda}=1$ (i.e. $\mathcal{N} \cap L^{2}=\emptyset$ ). Then there exist constants $C, K, k_{0}>0$ such that for any $\mathbf{k} \in \mathbb{R}^{3}$ with $k<k_{0}, j=1,2$ and any potential $B \in \mathcal{W}_{K}$ there exists a $\Phi_{j, \mathbf{k}}^{B} \in \mathcal{N}$ with

$$
\left\|\Phi_{j, \mathbf{k}}^{B}\right\| \leq C\left(\inf _{\Phi \in \mathcal{N},\|\Phi\|=1}|\langle\Phi, B, \Phi\rangle|+|k|\right)^{-1}
$$

and

$$
\left\|\phi(A+B, j, \mathbf{k}, \cdot)-\Phi_{j, \mathbf{k}}^{B}\right\|_{\infty}<C\left(\|B\|_{1}+\|B\|_{\infty}\right)\left(\inf _{\Phi \in \mathcal{N},\|\Phi\|=1}|\langle\Phi, B, \Phi\rangle|+|k|\right)^{-1} .
$$


Remark 3.6 Note, that for any $\mathbf{k} \in \mathbb{R}^{3}$ there exist two linearly independent generalized eigenfunctions $\chi(j, \mathbf{k}, \cdot)$ and two linearly independent generalized eigenfunctions $\chi_{-E_{k}}$ of the free Dirac operator with energy $\pm E_{k}= \pm \sqrt{1+k^{2}}$. Using CPT-symmetry the Theorem is also valid for potentials $A$ which are "critical" in the sense that they have bound states or a resonance with energy -1 . It then gives estimates on the generalized eigenfunctions with negative energy (i.e. $j=3,4$ ) of course.

To make it easier to understand the statement of Theorem 3.4. let us restrict ourselves on potentials $B_{\mu}$ which can be written as $B_{\mu}=\mu B_{0}$ for some fixed potential $B_{0}$ and $\mu \in\left[-\mu_{0}, \mu_{0}\right] . B_{0}$ and $\mu_{0} \in \mathbb{R}^{+}$ are chosen such, that $B_{\mu} \in \mathcal{W}_{K}$ for all $\mu \in\left[-\mu_{0}, \mu_{0}\right]$. Under these restrictions we get

Corollary 3.7 Let $A \in \mathcal{C}$ with $\bar{\lambda}=0$. Let $B_{0} \in L^{\infty} \cap L^{1}$ with $\left\langle\Phi, B_{0}, \Phi\right\rangle \neq 0$ for all $\Phi \in \mathcal{N} \backslash\{0\}$. Then there exist constants $C, \mu_{0}, k_{0}>0$ and constants $\gamma_{l}, l=1, \ldots, n=\operatorname{dim} \mathcal{N}$ such that for any $\mathbf{k} \in \mathbb{R}^{3}$ with $k<k_{0}, j=1,2$, any $\mu \in\left[-\mu_{0}, \mu_{0}\right]$ there exists a $\Phi_{j, \mathbf{k}}^{\mu} \in \mathcal{N}$ with

$$
\left\|\Phi_{j, \mathbf{k}}^{\mu}\right\| \leq C+C k \sum_{l=1}^{n}\left(\left|\mu+\gamma_{l} k^{2}\right|+k^{3}\right)^{-1}
$$

and

$$
\left\|\phi\left(A+\mu B_{0}, j, \mathbf{k}, \cdot\right)-\Phi_{j, \mathbf{k}}^{\mu}\right\|_{\infty}<C .
$$

Proof: We choose $\mu_{0}$ such that $\mu B_{0} \in \mathcal{W}_{K}$ for all $\mu \in\left[-\mu_{0}, \mu_{0}\right]$. Hence the assumptions of Theorem 3.4 hold for $\mu_{0}$ small enough and we only need to show that the right hand sides of (19) and (20) are bounded by the right hand sides of (23) and (24) respectively.

For that note that under the given assumptions

$$
\left\|\mu B_{0}\right\|_{1} \leq C k+2\left\|\mu B_{0}\right\|_{1}\left(\inf _{\Psi \in \mathcal{N},\|\Psi\|=1}\left\|\left(P_{\mathcal{N}}^{\|} \mu B_{0}+\widehat{R} k^{2}\right) \Psi\right\|+k^{3}\right)\left(\inf _{\Psi \in \mathcal{N},\|\Psi\|=1}\left|\left\langle\Psi, \mu B_{0}, \Psi\right\rangle\right|\right)^{-1}
$$

for appropriate $C<\infty$. This one can prove by considering two different cases. First assume that $\inf _{\Psi \in \mathcal{N},\|\Psi\|=1}\left|\left\langle\Phi, \mu B_{0}, \Phi\right\rangle\right|>2\|\widehat{R}\|_{o p} k^{2}$. It follows that

$$
\left(\inf _{\Psi \in \mathcal{N},\|\Psi\|=1}\left\|\left(P_{\mathcal{N}}^{\|} \mu B_{0}\right) \Psi\right\|\right)+k^{3} \leq\left(\inf _{\Psi \in \mathcal{N},\|\Psi\|=1}\left|\left\langle\Psi, \mu B_{0}, \Psi\right\rangle\right|\right) \leq \frac{1}{2} \inf _{\Psi \in \mathcal{N},\|\Psi\|=1}\left|\left\langle\Psi, \mu B_{0}, \Psi\right\rangle\right|
$$

and the second summand of (25) gives an appropriate bound. Assuming that $\inf _{\Psi \in \mathcal{N},\|\Psi\|=1}\left|\left\langle\Phi, \mu B_{0}, \Phi\right\rangle\right|<$ $2\|\widehat{R}\|_{o p} k^{2}$ we have for $\mu B_{0} \in \mathcal{W}_{K}$ (c.f. Definition 3.1) that $\left\|\mu B_{0}\right\|_{1}^{2}<2 K\|\widehat{R}\|_{o p} k^{2}$ and thus the first summand of (25) gives an appropriate bound.

Similarly one gets that

$$
\begin{aligned}
& C k\left(\left\|\mu B_{0}\right\|_{1}+\left\|\mu B_{0}\right\|_{\infty}\right)\left(\inf _{\Psi \in \mathcal{N},\|\Psi\|=1}\left\|\left(P_{\mathcal{N}}^{\|} \mu B_{0}+\widehat{R} k^{2}\right) \Psi\right\|+k^{3}\right)^{-1} \\
& <C\left(\left\|\mu B_{0}\right\|_{1}+\left\|\mu B_{0}\right\|_{\infty}\right)\left(\inf _{\Psi \in \mathcal{N},\|\Psi\|=1} \mid\left\langle\Psi, \mu B_{0}, \Psi\right\rangle\right)^{-1} .
\end{aligned}
$$

Assuming that $\inf _{\Psi \in \mathcal{N},\|\Psi\|=1}\left|\left\langle\Phi, \mu B_{0}, \Phi\right\rangle\right|>2\|\widehat{R}\|_{o p} k^{2}$ the formula can be proven as (25) above, assuming that $\inf _{\Psi \in \mathcal{N},\|\Psi\|=1}\left|\left\langle\Phi, \mu B_{0}, \Phi\right\rangle\right|<2\|\widehat{R}\|_{o p} k^{2}$ we have

$$
\begin{aligned}
& C k\left(\left\|\mu B_{0}\right\|_{1}+\left\|\mu B_{0}\right\|_{\infty}\right)\left(\inf _{\Psi \in \mathcal{N},\|\Psi\|=1}\left\|\left(P_{\mathcal{N}}^{\|} \mu B_{0}+\widehat{R} k^{2}\right) \Psi\right\|+k^{3}\right)^{-1}<C\left(\left\|\mu B_{0}\right\|_{1}+\left\|\mu B_{0}\right\|_{\infty}\right) k^{-3} \\
& =C\left(\left\|\mu B_{0}\right\|_{1}+\left\|\mu B_{0}\right\|_{\infty}\right) k^{-2} \leq C\left(\left\|\mu B_{0}\right\|_{1}+\left\|\mu B_{0}\right\|_{\infty}\right)\left(\inf _{\Psi \in \mathcal{N},\|\Psi\|=1}\left|\left\langle\Psi, \mu B_{0}, \Psi\right\rangle\right|\right)^{-1} \leq C,
\end{aligned}
$$

where we used in the last step that $\left(\left\|B_{0}\right\|_{1}+\left\|B_{0}\right\|_{\infty}\right)\left(\inf _{\Psi \in \mathcal{N},\|\Psi\|=1}\left|\left\langle\Psi, B_{0}, \Psi\right\rangle\right|\right)^{-1}$ exists by assumptions on $B_{0}$. With that and (20) equation (24) follows. 
Next we prove (23). Using (25) in (19), noting that

$$
\left\|\mu B_{0}\right\|_{1}\left(\inf _{\Psi \in \mathcal{N},\|\Psi\|=1}\left|\left\langle\Psi, \mu B_{0}, \Psi\right\rangle\right|\right)^{-1}=\left\|B_{0}\right\|_{1}\left(\inf _{\Psi \in \mathcal{N},\|\Psi\|=1}\left|\left\langle\Psi, B_{0}, \Psi\right\rangle\right|\right)^{-1}<C
$$

we have

$$
\left\|\Phi_{j, \mathbf{k}}^{\mu}\right\| \leq C+C k\left(\inf _{\Phi \in \mathcal{N},\|\Phi\|=1}\left\|\left(P_{\mathcal{N}}^{\|} \mu B_{0}+\widehat{R} k^{2}\right) \Phi\right\|+k^{3}\right)^{-1} .
$$

Recall that $\left\langle\Phi, B_{0}, \Phi\right\rangle \neq 0$ for all $\Phi \in \mathcal{N} \backslash\{0\}$, hence in particular $B \Phi \neq 0$ for all $\Phi \in \mathcal{N} \backslash\{0\}$. Thus the matrix $\widehat{B}_{0}: \mathcal{N} \rightarrow \mathcal{N}$ defined by

$$
\widehat{B}_{0} \Phi=P_{\mathcal{N}} B \Phi
$$

is invertible. Hence we get for (27)

$$
\begin{aligned}
\left\|\Phi_{j, \mathbf{k}}^{B}\right\| & \leq C+C k\left(\inf _{\Phi \in \mathcal{N},\|\Phi\|=1}\left\|\widehat{B}_{0}\left(\mu+\widehat{B}_{0}^{-1} \widehat{R} k^{2}\right) \Phi\right\|+k^{3}\right)^{-1} \\
& \leq C+C k\left\|\widehat{B}_{0}^{-1}\right\|_{o p}\left(\inf _{\Phi \in \mathcal{N},\|\Phi\|=1}\left\|\left(\mu+\widehat{B}_{0}^{-1} \widehat{R} k^{2}\right) \Phi\right\|+k^{3}\right)^{-1} .
\end{aligned}
$$

Using that $\left\|\widehat{B}_{0}^{-1}\right\|_{o p}<\infty$ and defining the symmetric operator $\widehat{M}: \mathcal{N} \rightarrow \mathcal{N}$ and the antisymmetric operator $\widehat{N}: \mathcal{N} \rightarrow \mathcal{N}$ by

$$
\widehat{M}:=\frac{1}{2}\left(\widehat{B}_{0}^{-1} \widehat{R}+\widehat{R} \widehat{B}_{0}^{-1}\right)
$$

and

$$
\widehat{N}:=\frac{1}{2}\left(\widehat{B}_{0}^{-1} \widehat{R}-\widehat{R} \widehat{B}_{0}^{-1}\right)
$$

one gets

$$
\begin{aligned}
\left\|\Phi_{j, \mathbf{k}}^{B}\right\| & \leq C+C k\left(\inf _{\Phi \in \mathcal{N},\|\Phi\|=1}\left\|\left(\mu+\widehat{M} k^{2}+\widehat{N} k^{2}\right) \Phi\right\|+k^{3}\right)^{-1} \\
& \leq C+C k\left(\inf _{\Phi \in \mathcal{N},\|\Phi\|=1}\left|\left\langle\Phi,\left(\mu+\widehat{M} k^{2}+\widehat{N} k^{2}\right) \Phi\right\rangle\right|+k^{3}\right)^{-1} .
\end{aligned}
$$

Note that for symmetric $\widehat{M}$ the $\left\langle\Phi,\left(\mu+\widehat{M} k^{2}\right) \Phi\right\rangle$ is real, whereas for antisymmetric $\widehat{N}$ the $\left\langle\Phi, \widehat{N} k^{2} \Phi\right\rangle$ is imaginary, hence

$$
\left\|\Phi_{j, \mathbf{k}}^{B}\right\| \leq C+C k\left(\inf _{\Phi \in \mathcal{N},\|\Phi\|=1}\left|\left\langle\Phi,\left(\mu+\widehat{M} k^{2}\right) \Phi\right\rangle\right|+k^{3}\right)^{-1}
$$

Let now $\left\{\Phi_{l}: l=1, \ldots, n=\operatorname{dim} \mathcal{N}\right\}$ be an orthonormal eigenbasis of $\mathcal{M}$, let $\gamma_{l}: l=1, \ldots, n$ be the respective eigenvalues. Note that the minimum of $\left|\left\langle\Phi,\left(\mu+\widehat{M} k^{2}\right) \Phi\right\rangle\right|$ is always realized for an eigenstate of $\mu+\widehat{M} k^{2}$, thus an element of $\left\{\Phi_{l}\right\}$. Which element will in general depend on $k$ and $\mu$, thus we have

$$
\begin{aligned}
\left\|\Phi_{j, \mathbf{k}}^{B}\right\| & \leq C+C k\left(\inf _{\Phi_{l}}\left|\left\langle\Phi_{l},\left(\mu+\widehat{M} k^{2}\right) \Phi_{l}\right\rangle\right|+k^{3}\right)^{-1} \\
& \leq C+C k \sum_{l=1}^{n}\left(\left|\mu+\gamma_{l} k^{2}\right|+k^{3}\right)^{-1}
\end{aligned}
$$




\section{Discussion of the Result}

Before proving the Theorem let us shortly clarify the physical meaning of Corollary 3.7 on a heuristic level.

(a) If $\bar{\lambda}=0$ it may happen that the nominator in the right hand side of (23) is of order $k^{3}$ (namely if $\mu+\gamma_{l} k^{2}=0$ for some $1 \leq l \leq n$ ). The respective $k$ 's where this happens are usually called "resonances of the potential $A+B$ " in the physics literature. Around the resonance the generalized eigenfunctions are of order $k^{-2}$.

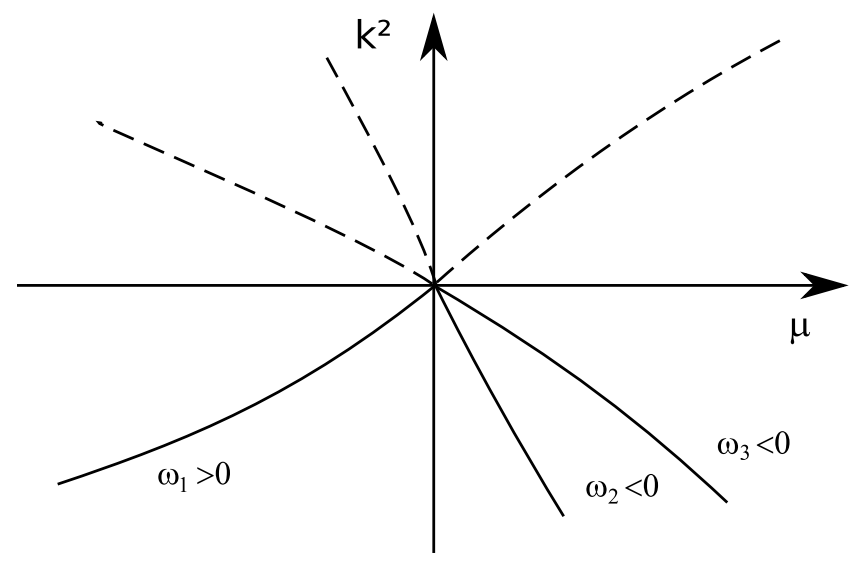

Figure 1: Bound states and Resonances (Illustration of (b)) The figure illustrates the position of bound states (illustrated by lines, $k$ is imaginary hence $k^{2}$ negative) and resonances (dashed lines, $k$ is real hence $k^{2}$ positive) of the Dirac operator $D=D^{0}+A+\mu B_{0}$, with $A, B_{0}$ and $\mu$ as in Corollary 3.7 .

(b) The results of Corollary 3.7 can also be used to roughly estimate the energy of bound states of "almost-critical" potentials $A+B$. Due to Lemma 2.2 bound states have energies smaller than 1 , so the respective $k=i \kappa$ is imaginary. Instead of (9) they satisfy (11), implying that $\left\|\phi_{E_{k}}\right\|_{\infty}=\infty$ for the respective $B$ and (imaginary) $k$ as one can see as follows. Heuristically speaking: "Normalize" (9), i.e. divide (9) on both sides by $\left\|\phi_{E_{k}}\right\|_{\infty}$. It follows that (9) and (11) are equivalent if (and only if) the right hand side of (9) divided by $\left\|\phi_{E_{k}}\right\|_{\infty}$ is equal to zero, hence if $\left\|\phi_{E_{k}}\right\|_{\infty}=\infty$.

Hence the energy $E_{k}$ of the $l^{\text {th }}$ bound state of the potential $A+B$ satisfies $\mu+\gamma_{l} k^{2} \approx 0$ if $\bar{\lambda}=0$ (see solid lines in figure (4). This implies, that bound states occur only if the respective $\mu$ and $\gamma_{l}$ have different sign. They "live" on different lines with slope $\gamma_{l}$ through the origin in the $k^{2}\left(\approx E_{k}-1\right)$ against $\mu$-plot (see figure 4).

This estimation is in line with the results of Theorem 1.1 by Klaus (in Klaus' Paper a plays the role of $\lambda$ ) concerning the behavior of the bound state energies at the threshold: $a=0 \Leftrightarrow \sigma:=\mu \sim$ $\kappa^{2} \Leftrightarrow E=\kappa^{2}$ is not analytic in $\sigma$ (since the next term in the power series is of order $\kappa^{3} \propto \sigma^{\frac{3}{2}}$ which destroys analyticity); $a \neq 0 \Leftrightarrow \sigma:=\mu \sim \kappa \Leftrightarrow E=\kappa^{2}$ is analytic in $\sigma$.

This idea is also helpful to find out the sign of the respective $\gamma_{l}$ : If $B_{0}$ is such, that there exist (don't exist) bound states with energy $E_{\kappa}$ for positive $\mu$ with $\mu-\gamma_{l} \kappa^{2} \approx 0$, the respective $\gamma_{l}$ is positive (negative) (see again figure 4).

There is physics in this: The fact, that there are bound states "living" on different lines comes from the fact, that adding the potential $B_{0}$ may destroy the degeneracy of $A$ (For example, if $A$ was purely electric, thus (at least) spin-degenerated, adding a small vector potential $B_{0}$ will in general destroy spin degeneracy). The degeneracy of the new bound states on each of these "lines" is equal to the multiplicity of the respective $\gamma_{l}$.

It follows, that also the "resonances" loose - at least partially - their degeneracy when a general potential $B_{0}$ is added. The estimates (concerning the sum) in Corollary 3.7 reflect this fact: Each 
summand represents a "resonance". In this sense one can heuristically guess that the generalized eigenfunction is of leading order equal to

$$
\phi(A+B, j, \mathbf{k}, \cdot) \approx \sum_{p=1}^{n} \frac{C k}{\mu+\gamma_{l} k^{2}+i C_{3} k^{3}} \Phi_{p},
$$

where the set $\left\{\Phi_{p}: 1 \leq p \leq 1\right\}$ is a basis of $\mathcal{N}$.

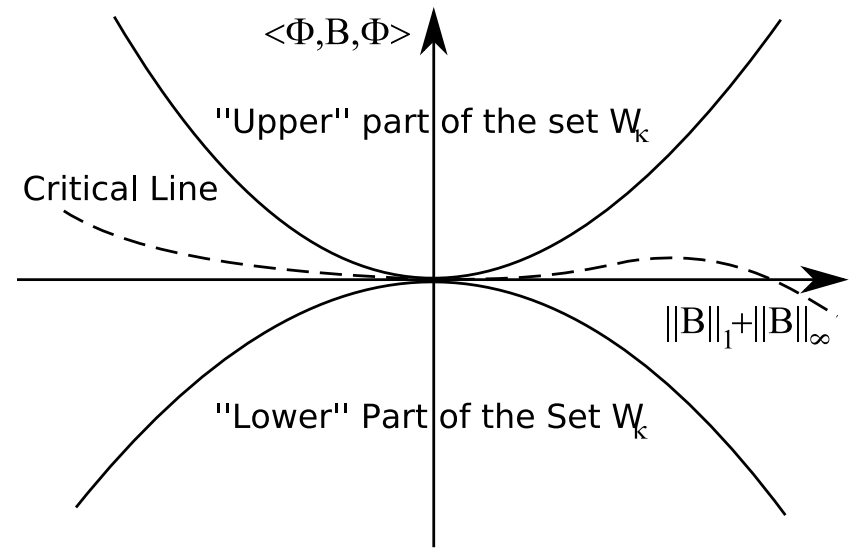

Figure 2: Illustration of the Set $\mathcal{W}_{K}$ for $n=1$ and normalized $\Phi$

$\mathcal{W}_{K}$ lies inside two parabolas and is the region where the Theorem applies. On the "Critical Line" are potentials with critical $A+B$ (see (c)). Both parabolas and the "critical line" touch in the origin, implying heuristically that $\langle\Phi, B, \Phi\rangle=O\left(\left(\|B\|_{1}+\|B\|_{\infty}\right)^{2}\right)$ for critical $A+B$.

Due to part (b) one of these two parabolas contains potentials which have bound states, the other one contains potentials which have "resonances" (which one, depends on $A$. For positive, purely electric $A$ on can show that the potentials in the lower "parabola" have bound states ).

(c) Let us explain the meaning of the set $\mathcal{W}_{K}$ (see figure 4 ).

Let $A \in \mathcal{C}$. Disturbing $A$ by a small short range potential $B$ it may happen, that $A+B$ stays critical.

If $A+B$ stays critical, the result of Jensen and Kato gives us, that the respective generalized eigenfunctions diverge for $k=0$. Looking at (19) it follows that in this case either $\langle\Phi, B, \Phi\rangle=0$ for some $\Phi \in \mathcal{N}$ or that the requirements of Theorem 3.4 are not satisfied, which means that $B \notin \mathcal{W}_{K}$. This fact is a strong requirement on $B$ for the criticality of $A+B$ (see figure 4) for the non-degenerate case. Remember that by definition $3.1 B \in \mathcal{W}_{K} \Leftrightarrow \frac{\left(\|B\|_{1}+\|B\|_{\infty}\right)^{2}}{|\langle\Phi, B, \Phi\rangle|} \leq K$ for all normalized $\Phi \in \mathcal{N} \Leftrightarrow$ $|\langle\Phi, B, \Phi\rangle| \geq K^{-1}\left(\|B\|_{1}+\|B\|_{\infty}\right)^{2}$. So in the $\langle\Phi, B, \Phi\rangle$ against $\|B\|_{1}+\|\left.\mathcal{B}\right|_{\infty}$ plot, $\mathcal{W}_{K}$ lies inside two parabolas with curvature $\pm K$ (see figure 4).

\section{Proof of the Theorem}

The set $\mathcal{M}^{\perp}$ has the interesting property that it is invariant under $T_{1}^{A}$, a fact which will play a crucial role in what follows

Lemma 5.1 For any $A \in \mathcal{C}$ we have that

$$
h^{\perp} \in \mathcal{M}^{\perp} \Leftrightarrow T_{1}^{A} h^{\perp} \in \mathcal{M}^{\perp} .
$$


Proof: We show first that for $h, g \in \mathcal{B}$ and $A, B \in L_{1}$

$$
\left\langle h, A, T_{E}^{B} g\right\rangle=\left\langle T_{E}^{A} h, B, g\right\rangle
$$

by computing

$$
\begin{aligned}
\left\langle h, A, T_{E}^{B} g\right\rangle & =\int h^{\dagger}(\mathbf{x}) A(\mathbf{x}) T_{E}^{B} g(\mathbf{x}) d^{3} x \\
& =\int h^{\dagger}(\mathbf{x}) A(\mathbf{x}) \int G_{E}^{+}(\mathbf{x}-\mathbf{y}) B(\mathbf{y}) g(\mathbf{y}) d^{3} y d^{3} x \\
& =\iint h^{\dagger}(\mathbf{x}) A(\mathbf{x}) G_{E}^{+}(\mathbf{x}-\mathbf{y}) d^{3} x B(\mathbf{y}) g(\mathbf{y}) d^{3} y \\
& =\int\left(T_{E_{k}}^{A} h\right)^{\dagger}(\mathbf{y}) B(\mathbf{y}) g(\mathbf{y}) d^{3} y \\
& =\left\langle T_{E}^{A} h, B, g\right\rangle .
\end{aligned}
$$

We may apply this to $h \in \mathcal{B}$ and $g=\Phi \in \mathcal{N}$ to obtain

$$
\langle h, A, \Phi\rangle=\left\langle h, A, T_{1}^{A} \Phi\right\rangle=\left\langle T_{1}^{A} h, A, \Phi\right\rangle .
$$

This equation directly implies the Lemma: If $h \in \mathcal{M}^{\perp}$ (which means that $\langle h, A, \Phi\rangle=0$ ) it follows that $T_{1}^{A} h \in \mathcal{M}^{\perp}$ (which means $\left\langle T_{1}^{A} h, A, \Phi\right\rangle=0$ ) and vice versus.

Furthermore we have

Lemma 5.2 Using the definitions above we have

$$
\mathcal{B}=\mathcal{M}^{\|} \oplus \mathcal{M}^{\perp}
$$

$$
\mathcal{B}=\mathcal{N} \oplus \mathcal{M}^{\perp}
$$

Remark 5.3 Note that $\langle A \Phi, A, \Phi\rangle>0$, hence $\mathcal{M}^{\|} \cap \mathcal{M}^{\perp}=\{0\}$.

Using that $\mathcal{M}^{\|} \cap \mathcal{M}^{\perp}=\{0\}$, part (a) of the Lemma defines projectors $P_{\mathcal{M}}^{\|}$and $P_{\mathcal{M}}^{\perp}$ with $P_{\mathcal{M}}^{\|} \mathcal{B} \subset \mathcal{M}^{\|}$, $P_{\mathcal{M}}^{\perp} \mathcal{B} \subset \mathcal{M}^{\perp}$ and $P_{\mathcal{M}}^{\|}+P_{\mathcal{M}}^{\perp}=1$

Using that $\mathcal{N} \cap \mathcal{M}^{\perp}=\{0\}$ (see Definition [3.2), (b) defines projectors $P_{\mathcal{N}}^{\|}$and $P_{\mathcal{N}}^{\perp}$ with $P_{\mathcal{N}}^{\|} \mathcal{B} \subset \mathcal{N}$, $P_{\mathcal{N}}^{\perp} \mathcal{B} \subset \mathcal{M}^{\perp}$ and $P_{\mathcal{N}}^{\|}+P_{\mathcal{N}}^{\perp}=1$.

Proof (a), (b) " $\supset$ " Since $\mathcal{M}^{\|}, \mathcal{M}^{\perp}, \mathcal{N} \subset \mathcal{B}$ it follows that $\mathcal{B} \supset \mathcal{M}^{\|} \oplus \mathcal{M}^{\perp}$ and $\mathcal{B} \supset \mathcal{N}^{\|} \oplus \mathcal{M}^{\perp}$.

(a) "C" Let $f \in \mathcal{B},\left\{\Phi_{p}\right\} p=1 \ldots n$ be a basis of $\mathcal{N}$. Define the vector $\vec{f} \in \mathbb{R}^{n}$ by $f_{p}:=\left\langle f, A, \Phi_{p}\right\rangle$ and for any $q=1 \ldots n$ the vector $\vec{\Phi}^{q}$ by $\Phi_{p}^{q}:=\left\langle A \Phi_{q}, A, \Phi_{p}\right\rangle$.

We will show by contradiction that the $\vec{\Phi}^{q}$ are linearly independent. Assume that the vectors $\vec{\Phi}^{q}$ are linearly dependent, i.e. that it is possible to find non-trivial complex numbers $\gamma_{q}, q=1 \ldots n$ such that $0=\sum_{q=1}^{n} \gamma_{j} \vec{\Phi}^{q}$. In other words $\left\langle A \sum_{q=1}^{n} \gamma_{q} \Phi_{q}, A, \Phi_{p}\right\rangle=0$ for all $p=1 \ldots n$, hence $A \sum_{q=1}^{n} \gamma_{q} \Phi_{q} \in \mathcal{M}^{\perp}$. Furthermore we have by definition of $\mathcal{M}^{\|}$that $A \sum_{q=1}^{n} \gamma_{q} \Phi_{q} \in \mathcal{M}^{\|}$. Hence $\mathcal{M}^{\|} \cap \mathcal{M}^{\perp}=\{0\}$ implies $A \sum_{q=1}^{n} \gamma_{q} \Phi_{q} \equiv 0$ (and thus $\sum_{q=1}^{n} \gamma_{q} \Phi_{q}=0$ on the support of $A$ ). But the only eigenfunction which is equal to zero on the support of $A$ is $\Phi \equiv 0$, hence $\sum_{q=1}^{n} \gamma_{q} \Phi_{q} \equiv 0$. This contradicts to the fact that the $\Phi_{q}$ are linearly independent.

It follows that the vectors $\vec{\Phi}^{q}$ are linearly independent and thus they form a Basis of $\mathbb{C}^{n}$, hence we can find complex numbers $\gamma_{q}, q=1 \ldots n$ such that $\sum_{q=1}^{n} \gamma_{q} \vec{\Phi}^{q}=\vec{f}$. Defining $f^{\|}:=A \sum_{q=1}^{n} \gamma_{q} \Phi^{q} \in$ $\mathcal{M}^{\|}$it follows that $\left\langle f, A, \Phi_{p}\right\rangle=\left\langle f^{\|}, A, \Phi_{p}\right\rangle$ for any $p=1 \ldots n$, i.e. $f^{\perp}:=f-f^{\|} \in \mathcal{M}^{\perp}$. 
(b) "C" This proof is equivalent to (a) "C". Define $\vec{f} \in \mathbb{R}^{n}$ by $f_{p}:=\left\langle f, A, \Phi_{p}\right\rangle$ and for any $q=1 \ldots n$ a vector $\vec{\Phi}^{q}$ by $\Phi_{p}^{q}:=\left\langle\Phi_{q}, A, \Phi_{p}\right\rangle$. Under the assumption that the $\vec{\Phi}^{q}$ are linearly dependent we have the existence of non trivial $\gamma_{q}$ such that $\left\langle\sum_{q=1}^{n} \gamma_{q} \Phi_{q}, A, \sum_{p=1}^{n} \Phi_{p}\right\rangle=0$ for any $p=1 \ldots n$. Since $\mathcal{N} \cap \mathcal{M}^{\perp}=\{0\}$ it follows that $\sum_{q=1}^{n} \gamma_{q} \Phi_{q} \equiv 0$ which contradicts to the linear independence of the $\Phi_{q}$.

It follows that the $\vec{\Phi}^{q}$ are linearly independent, hence we can find complex numbers $\gamma_{q}, q=1 \ldots n$ such that $\sum_{q=1}^{n} \gamma_{j} \vec{\Phi}^{q}=\vec{f}$. Defining $f^{\|}:=\sum_{q=1}^{n} \gamma_{q} \Phi^{q} \in \mathcal{N}$ it follows that $\left\langle f, A, \Phi_{p}\right\rangle=\left\langle f^{\|}, A, \Phi_{p}\right\rangle$ for any $p=1 \ldots n$, i.e. $f^{\perp}:=f-f^{\|} \in \mathcal{M}^{\perp}$.

We now arrive at the main Lemma.

Lemma 5.4 Let $A \in \mathcal{C}$. Then there exist constants $C, C^{\prime}>0$, a selfadjoint sesquilinear map $r: \mathcal{N} \times \mathcal{N} \rightarrow$ $\mathbb{C}$ and a anti-selfadjoint sesquilinear map $s: \mathcal{N} \times \mathcal{N} \rightarrow \mathbb{C}$ with $s(\chi, \chi) \neq 0$ for all $\chi \in \mathcal{N}$ such that for any $\mathbf{k} \in \mathbb{R}^{3}$ with $k<1$, any potential $B$ with $B \in L^{1} \cap L^{\infty}$, any normalized $m^{\perp} \in \mathcal{M}^{\perp}$ and any normalized $\Phi, \Psi \in \mathcal{N}$

(a)

$$
\left|\left\langle\Phi, A,\left(1-T_{E_{k}}^{A+B}\right) m^{\perp}\right\rangle\right|<C\left(\|A\|_{1}+\|B\|_{1}\right)\left(\bar{\lambda} k+k^{2}\right),
$$

$$
\left\|P_{\mathcal{M}}^{\perp}\left(1-T_{E_{k}}^{A+B}\right) m^{\perp}\right\|_{\infty} \geq C-C^{\prime}\left(\bar{\lambda} k+k^{2}+\|B\|_{1}+\|B\|_{\infty}\right)
$$

$$
\left\|P_{\mathcal{M}}^{\perp}\left(1-T_{E_{k}}^{A+B}\right) \Phi\right\|_{\infty}<C\left(\bar{\lambda} k+k^{2}+\|B\|_{1}+\|B\|_{\infty}\right)
$$

$$
\begin{aligned}
\left\langle\Phi, A,\left(1-T_{E_{k}}^{A+B}\right) \Psi\right\rangle= & \langle\Phi, B, \Psi\rangle+r(\Phi, \Psi) k^{2} \\
& +i r(\Phi, \lambda(\Psi)) k+s(\Phi, \Psi) k^{3}+o\left(k^{3}\right)+\|B\|_{1}\left(\bar{\lambda} \mathcal{O}(k)+\mathcal{O}\left(k^{2}\right)\right) .
\end{aligned}
$$

\section{Proof:}

The proof is given in the Appendix.

Using this Lemma we can estimate the inverse of $1-T_{E_{k}}^{A+B}$.

Lemma 5.5 Let $A \in \mathcal{C}$. Then there exist constants

$C, C^{\prime}, K, k_{0}>0, C_{0}, C_{1} \in \mathbb{R}$, a selfadjoint sesquilinear map $r: \mathcal{N} \times \mathcal{N} \rightarrow \mathbb{C}$ and an anti-selfadjoint sesquilinear map $s: \mathcal{N} \times \mathcal{N} \rightarrow \mathbb{C}$ with $s(\chi, \chi) \neq 0$ for all $\chi \in \mathcal{N}$ such that for any normalized $\Phi \in \mathcal{N}$, any $\mathbf{k} \in \mathbb{R}^{3}$ with $k<k_{0}$, any potential $B \in \mathcal{W}_{K}$ there exists a normalized $\Psi \in \mathcal{N}$ such that

$$
\left\|P_{\mathcal{N}}^{\|}\left(1-T_{E_{k}}^{A+B}\right)^{-1}(A \Phi)\right\| \leq C\left(\sup _{\chi \in \mathcal{N},\|\chi\|=1}\left|\langle\chi, B, \Psi\rangle+r(\chi, \Psi) k^{2}-i k\langle\chi, A, \lambda(\Psi)\rangle+s(\chi, \Psi) k^{3}\right|\right)^{-1}
$$

and

$$
\begin{aligned}
& \left\|P_{\mathcal{N}}^{\perp}\left(1-T_{E_{k}}^{A+B}\right)^{-1}(A \Phi)\right\|_{\infty} \leq C\left(\bar{\lambda} k+k^{2}+\|B\|_{1}+\|B\|_{\infty}\right) \\
& \left(\sup _{\chi \in \mathcal{N},\|\chi\|=1}\left|\langle\chi, B, \Psi\rangle+r(\chi, \Psi) k^{2}-i k\langle\chi, A, \lambda(\Psi)\rangle+s(\chi, \Psi) k^{3}\right|\right)^{-1} .
\end{aligned}
$$

Furthermore we have that for any normalized $m^{\perp} \in \mathcal{M}^{\perp}$ there exists a normalized $\Psi \in \mathcal{N}$ such that

$$
\begin{aligned}
& \left\|P_{\mathcal{N}}^{\|}\left(1-T_{E_{k}}^{A+B}\right)^{-1} m^{\perp}\right\|_{\infty} \\
& \leq C\left|\bar{\lambda} k+k^{2}\right|\left(\sup _{\chi \in \mathcal{N},\|\chi\|=1}\left|\langle\chi, B, \Psi\rangle+r(\chi, \Psi) k^{2}-i k\langle\chi, A, \lambda(\Psi)\rangle+s(\chi, \Psi) k^{3}\right|\right)^{-1}
\end{aligned}
$$


and

$$
\left\|P_{\mathcal{N}}^{\perp}\left(1-T_{E_{k}}^{A+B}\right)^{-1} m^{\perp}\right\|_{\infty} \leq C .
$$

Proof: Let $A \in \mathcal{C}$. Choose $k_{0}$ and $K$ (there will be further restrictions on $k_{0}$ and $K$ below, so the final $k_{0}$ and $K$ may at the end be smaller) such that there exists a $C>0$ such that

$$
\left\|P_{\mathcal{M}}^{\perp}\left(1-T_{E_{k}}^{A+B}\right) h^{\perp}\right\|_{\infty} \geq C
$$

for any $h^{\perp} \in \mathcal{M}^{\perp}$, any $\mathbf{k} \in \mathbb{R}^{3}$ with $k<k_{0}$ and any potential $B \in \mathcal{W}_{K}$ (in view of Lemma 5.4 (b) such a choice is possible).

Then (using Lemma $5.4(\mathrm{a}),(\mathrm{c})$ and $(\mathrm{d})$ ) one can find a constant $C>0$ such that for any $\mathbf{k} \in \mathbb{R}^{3}$ with $k<k_{0}$, any potential $B \in \mathcal{W}_{K}$ (i.e. bounded $\|B\|_{1}$ ) and any normalized $\widetilde{\Phi} \in \mathcal{N}, m^{\perp} \in \mathcal{M}^{\perp}$

$$
\begin{gathered}
\sup _{\chi \in \mathcal{N},\|\chi\|=1}\left|\left\langle\chi, A,\left(1-T_{E_{k}}^{A+B}\right) m^{\perp}\right\rangle\right|<C\left(\bar{\lambda} k+k^{2}\right)=: t_{1}, \\
\left\|P_{\mathcal{M}}^{\perp}\left(1-T_{E_{k}}^{A+B}\right) \Phi\right\|_{\infty}<C\left(\bar{\lambda} k+k^{2}+\|B\|_{1}+\|B\|_{\infty}\right)=: t_{2}
\end{gathered}
$$

and

$$
\begin{aligned}
& \sup _{\chi \in \mathcal{N},\|\chi\|=1}\left\langle\chi, A,\left(1-T_{E_{k}}^{A+B}\right) \Phi\right\rangle \\
& =\sup _{\chi \in \mathcal{N},\|\chi\|=1}\left|\langle\chi, B, \Phi\rangle-i k\langle\chi, A, \lambda(\Phi)\rangle+k^{2} r(\chi, \Phi)+k^{3} s(\chi, \Phi)\right| \\
& \quad+\mathrm{o}\left(k^{3}\right)+\mathcal{O}\left(\|B\|_{1}\right)\left(\bar{\lambda} \mathcal{O}(k)+\mathcal{O}\left(k^{2}\right)\right) .
\end{aligned}
$$

Next we will show that the first summand will suffice for our estimates, i.e. that there exists a constant $C>0$ such that

$$
\begin{aligned}
& \sup _{\chi \in \mathcal{N},\|\chi\|=1}\left|\left\langle\chi, A,\left(1-T_{E_{k}}^{A+B}\right) \Phi\right\rangle\right| \\
& \quad \geq C \sup _{\chi \in \mathcal{N},\|\chi\|=1}\left|\langle\chi, B, \Phi\rangle-i k\langle\chi, A, \lambda(\Phi)\rangle+k^{2} r(\chi, \Phi)+k^{3} s(\chi, \Phi)\right|=: t_{3} .
\end{aligned}
$$

Therefore we have to show that for sufficiently small $K, k_{0}$ :

$$
t_{3} \gg \mathrm{o}\left(k^{3}\right)+\mathcal{O}\left(\|B\|_{1}\right)\left(\bar{\lambda} \mathcal{O}(k)+\mathcal{O}\left(k^{2}\right)\right)
$$

which we will do next.

We will prove (38) for $\bar{\lambda}=1, \bar{\lambda}=0$ and $\|B\|_{1}=\mathcal{O}(k)$ and $\bar{\lambda}=0$ and $\|B\|_{1} \gg k$ separately.

$1^{\text {st }}$ Case: Assume that $\bar{\lambda}=1$. Then the leading order of $t_{3}$ is obviously greater than or equal to $\langle\Psi, B, \Psi\rangle-$ $i k\langle\Psi, A, \lambda(\Psi)\rangle$. The first summand is real, the second summand is (see (15))

$$
-i k \iint \Psi^{t}(\mathbf{x}) A(\mathbf{x})(1+\beta) A(\mathbf{y}) \Psi(\mathbf{y}) d^{3} y d^{3} x=-i k \lambda^{t}(\Psi)(1+\beta) \lambda(\Psi)
$$

and - since $\beta$ is selfadjoint - imaginary (and not equal to zero). Hence there exists a $C>0$ such that $\langle\Psi, B, \Psi\rangle-i k\langle\Psi, A, \lambda(\Psi)\rangle>C k$ and (38) holds.

$2^{\text {nd }}$ Case: Assume that $\bar{\lambda}=0$ and $\|B\|_{1}=\mathcal{O}\left(K^{-\frac{1}{2}} k\right)$. Similar as above there exists a $C>0$ such that $\langle\Psi, B, \Psi\rangle-k^{2} r(\chi, \Phi)-k^{3} s(\chi, \Phi)>C k^{3}$. Since in this case $\|B\|_{1} \mathcal{O}\left(k^{2}\right)=\mathcal{O}\left(k^{3}\right)$ equation (38) holds.

$3^{r d}$ Case: Assume that $\bar{\lambda}=0$ and $\|B\|_{1} \gg K^{-\frac{1}{2}} k$. Since $B \in \mathcal{W}_{K}$ it follows that $\langle\Psi, B, \Psi\rangle \gg k^{2}$, hence $\langle\Psi, B, \Psi\rangle-k^{2} r(\chi, \Phi) \gg k^{2}$ and (38) holds. 
We next prove (31) and (31). We define

$$
\begin{aligned}
\omega \Psi & :=P_{\mathcal{N}}^{\|}\left(1-T_{E_{k}}^{A+B}\right)^{-1}(A \Phi) \\
h^{\perp}(\mathbf{k}, B) & :=P_{\mathcal{N}}^{\perp}\left(1-T_{E_{k}}^{A+B}\right)^{-1}(A \Phi),
\end{aligned}
$$

with $\omega>0$ and $\|\Psi\|_{\infty}=1$.

It follows that

$$
\left(1-T_{E_{k}}^{A+B}\right)\left(\omega \Psi+h^{\perp}(\mathbf{k}, B)\right)=A \Phi
$$

hence

$$
\sup _{\chi \in \mathcal{N},\|\chi\|=1}\left|\left\langle\chi, A,\left(1-T_{E_{k}}^{A+B}\right)\left(\omega \Psi+h^{\perp}(\mathbf{k}, B)\right)\right\rangle\right|=\sup _{\chi \in \mathcal{N},\|\chi\|=1}|\langle\chi, A, A \Phi\rangle|:=C_{\Phi}
$$

and

$$
P_{\mathcal{M}}^{\perp}\left(1-T_{E_{k}}^{A+B}\right)\left(\omega \Psi+h^{\perp}(\mathbf{k}, B)\right)=0
$$

Using (37) and (35) we get

$$
t_{3} \omega<t_{1}\left\|h^{\perp}(\mathbf{k}, B)\right\|_{\infty}+C_{\Phi}
$$

using (36) and (34) we get

$$
t_{2}|\omega| \geq C\left\|h^{\perp}(\mathbf{k}, B)\right\|_{\infty}
$$

hence

$$
\begin{aligned}
t_{3} \omega & <t_{1} \frac{t_{2}}{C} \omega+C_{\Phi} \\
t_{3} \omega-t_{1} \frac{t_{2}}{C} \omega & <C_{\Phi} \\
\omega & <C_{\Phi}\left(t_{3}-\frac{t_{1} t_{2}}{C}\right)^{-1} .
\end{aligned}
$$

Note, that $C_{\Phi}$ is bounded uniformly in normalized $\Phi$. To get (31) it is left to show that for small enough $k_{0}, K$

$$
\frac{t_{1} t_{2}}{C}<\frac{t_{3}}{2}
$$

uniform in $k<k_{0}$ and $B \in \mathcal{W}_{K}$.

We will prove (42) for $\bar{\lambda}=1, \bar{\lambda}=0$ and $\|B\|_{1}+\|B\|_{\infty}=\mathcal{O}(\sqrt{K} k)$ and $\bar{\lambda}=0$ and $\|B\|_{1}+\|B\|_{\infty} \gg \sqrt{K} k$ separately.

$1^{\text {st }}$ Case: Assume that $\bar{\lambda}=1$. Then we have that $t_{1} t_{2}$ is of order $k\left(\bar{\lambda} k+k^{2}+\|B\|_{1}+\|B\|_{\infty}\right)$ and $\left|t_{3}\right|$ is of order $k$ (see above). Hence for $K$ small enough (i.e. $\|B\|_{1}$ and $\|B\|_{\infty}$ small enough) and $k_{0}$ small enough (42) follows.

$2^{\text {nd }}$ Case: Assume that $\bar{\lambda}=0$ and $\|B\|_{1}+\|B\|_{\infty}=\mathcal{O}(\sqrt{K} k)$. Then we have that $t_{3}$ is of order $k^{3}$ and $t_{1} t_{2}$ is of order $k^{2}\left(k^{2}+\|B\|_{1}+\|B\|_{\infty}\right)$. Hence for small enough $K$ (42) follows.

$3^{\text {rd }}$ Case: Assume that $\bar{\lambda}=0$ and $\|B\|_{1}+\|B\|_{\infty} \gg \sqrt{K} k$, i.e.

$$
\sup _{\chi \in \mathcal{N},\|\chi\|=1}|\langle\chi, B, \Phi\rangle| \gg k^{2} .
$$

It follows that $\sup _{\chi \in \mathcal{N},\|\chi\|=1} \mid\langle\chi, B, \Phi\rangle-k^{2} r(\chi, \Phi) \gg k^{2}$, hence $t_{3} \gg k^{2}$ and (42) follows.

In view of (31) and (41) we have that

$$
\left\|h^{\perp}(\mathbf{k}, B)\right\|_{\infty} \leq \frac{t_{2}}{C t_{3}}
$$


which is - in view of (36), (37) and (40) - exactly (31).

(32) and (33) can be verified in a similar way as (31) and (31). We define

$$
\begin{aligned}
\omega \Psi & :=P_{\mathcal{N}}^{\|}\left(1-T_{E_{k}}^{A+B}\right)^{-1}\left(m^{\perp}\right) \\
h^{\perp}(\mathbf{k}, B) & :=P_{\mathcal{N}}^{\perp}\left(1-T_{E_{k}}^{A+B}\right)^{-1}\left(m^{\perp}\right),
\end{aligned}
$$

with $\omega>0$ and $\|\Psi\|_{\infty}=1$.

It follows that

$$
\left(1-T_{E_{k}}^{A+B}\right)\left(\omega \Psi+h^{\perp}(\mathbf{k}, B)\right)=m^{\perp},
$$

hence

$$
\sup _{\chi \in \mathcal{N},\|\chi\|=1}\left|\left\langle\chi, A,\left(1-T_{E_{k}}^{A+B}\right)\left(\omega \Psi+h^{\perp}(\mathbf{k}, B)\right)\right\rangle\right|=0
$$

and

$$
\left\|P_{\mathcal{M}}^{\perp}\left(1-T_{E_{k}}^{A+B}\right)\left(\omega \Psi+h^{\perp}(\mathbf{k}, B)\right)\right\|_{\infty}=1 .
$$

Using (37) and (35) we get

$$
t_{3}|\omega|<t_{1}\left\|h^{\perp}(\mathbf{k}, B)\right\|_{\infty}
$$

using (36) and (34) we get

$$
1-t_{2}|\omega| \geq C\left\|h^{\perp}(\mathbf{k}, B)\right\|_{\infty}
$$

hence

$$
C\left\|h^{\perp}(\mathbf{k}, B)\right\|_{\infty} \leq 1-\frac{t_{1} t_{2}}{t_{3}}\left\|h^{\perp}(\mathbf{k}, B)\right\|_{\infty} .
$$

In view of (42) $\frac{t_{1} t_{2}}{t_{3}}<\frac{1}{2} C$. It follows that $\left\|h^{\perp}(\mathbf{k}, B)\right\|_{\infty}$ is of order one, which is exactly (33)).

In view of (33) and (45) we have that

$$
|\omega| \leq \frac{t_{1}}{C t_{3}}
$$

which is - in view of (36), (37) and (44) - exactly (31). $\bar{\lambda}=0$

The Lemma can be written in a much nicer way, separating the different cases $\bar{\lambda}=0$ and $\bar{\lambda}=1$. For

Corollary 5.6 Let $A \in \mathcal{C}$ with $\bar{\lambda}=0$ (i.e. $\mathcal{N} \subset L^{2}$ ). Then there exist constants $C, K, k_{0}>0$ and a selfadjoint linear map $\widehat{R}: \mathcal{N} \rightarrow \mathcal{N}$ such that for any normalized $\Phi \in \mathcal{N}$, any $\mathbf{k} \in \mathbb{R}^{3}$ with $k<k_{0}$, any potential $B \in \mathcal{W}_{K}$

$$
\left\|P_{\mathcal{N}}^{\|}\left(1-T_{E_{k}}^{A+B}\right)^{-1}(A \Phi)\right\| \leq C\left(\inf _{\Psi \in \mathcal{N},\|\Psi\|=1}\left\|\left(P_{\mathcal{N}}^{\|} B+\widehat{R} k^{2}\right) \Psi\right\|+k^{3}\right)^{-1}
$$

and

$$
\left\|P_{\mathcal{N}}^{\perp}\left(1-T_{E_{k}}^{A+B}\right)^{-1}(A \Phi)\right\|_{\infty} \leq C\left(k^{2}+\|B\|_{1}+\|B\|_{\infty}\right)\left(\inf _{\Psi \in \mathcal{N},\|\Psi\|=1}\left\|\left(P_{\mathcal{N}}^{\|} B+\widehat{R} k^{2}\right) \Psi\right\|+k^{3}\right)^{-1} .
$$

Furthermore we have for any normalized $m^{\perp} \in \mathcal{M}^{\perp}$

$$
\left\|P_{\mathcal{N}}^{\|}\left(1-T_{E_{k}}^{A+B}\right)^{-1} m^{\perp}\right\|_{\infty} \leq C k^{2}\left(\inf _{\Psi \in \mathcal{N},\|\Psi\|=1}\left\|\left(P_{\mathcal{N}}^{\|} B+\widehat{R} k^{2}\right) \Psi\right\|+k^{3}\right)^{-1}
$$

and

$$
\left\|P_{\mathcal{N}}^{\perp}\left(1-T_{E_{k}}^{A+B}\right)^{-1} m^{\perp}\right\|_{\infty} \leq C .
$$


Proof: The Corollary follows directly from Lemma 5.5 Note, that we consider the case $\mathcal{N} \subset L^{2}$, i.e. there exists a selfadjoint linear map $\widehat{R}: \mathcal{N} \rightarrow \mathcal{N}$ and a antiselfadjoint linear map $\widehat{S}: \mathcal{N} \rightarrow \mathcal{N}$ such that $\langle\Phi, \widehat{R} \chi\rangle=r(\Phi, \chi)$ and $\langle\Phi, \widehat{S} \chi\rangle=s(\Phi, \chi)$ for $r$ and $s$ coming from the Lemma. Recall that $s(\Phi, \Phi) \neq 0$ for all $\Phi \in \mathcal{N} \backslash\{0\}$, hence $\langle\Phi, S \Phi\rangle \neq 0$ for all $\Phi \in \mathcal{N} \backslash\{0\}$

Using this and $\bar{\lambda}=0$ we have

$$
\sup _{\chi \in \mathcal{N},\|\chi\|=1}\left|\langle\chi, B, \Psi\rangle+r(\chi, \Psi) k^{2}+s(\chi, \Psi) k^{3}\right|=\sup _{\chi \in \mathcal{N},\|\chi\|=1}\left|\left\langle\chi, B+\widehat{R} k^{2}+\widehat{S} k^{3}, \Psi\right\rangle\right| .
$$

Note, that

$$
\sup _{\chi \in \mathcal{N},\|\chi\|=1}\left|\left\langle\chi, B+\widehat{R} k^{2}+\widehat{S} k^{3}, \Psi\right\rangle\right| \geq\left|\left\langle\Psi, B+\widehat{R} k^{2}+\widehat{S} k^{3}, \Psi\right\rangle\right|
$$

Since $B$ and $\widehat{R}$ are selfadjoint and $\widehat{S}$ is anti-selfadjoint, the first two summands are real, the last is imaginary. Furthermore we have that $\langle\Phi, S \Phi\rangle \neq 0$ for all $\Phi \in \mathcal{N} \backslash\{0\}$. Hence there exists a constants $C \in \mathbb{R} \backslash\{0\}$ such that

$$
\sup _{\chi \in \mathcal{N},\|\chi\|=1}\left|\left\langle\chi, B+\widehat{R} k^{2}+\widehat{S} k^{3}, \Psi\right\rangle\right| \geq C k^{3} .
$$

Furthermore we have that

$$
\sup _{\chi \in \mathcal{N},\|\chi\|=1}\left|\left\langle\chi, B+\widehat{R} k^{2}+\widehat{S} k^{3}, \Psi\right\rangle\right| \geq \sup _{\chi \in \mathcal{N},\|\chi\|=1}\left|\left\langle\chi, B+\widehat{R} k^{2}, \Psi\right\rangle\right|-k^{3}\|\widehat{S}\|^{o p},
$$

hence with (53) there exists a $C>0$ such that

$$
\begin{aligned}
C \sup _{\chi \in \mathcal{N},\|\chi\|=1}\left|\left\langle\chi, B+\widehat{R} k^{2}+\widehat{S} k^{3}, \Psi\right\rangle\right| & \geq \sup _{\chi \in \mathcal{N},\|\chi\|=1}\left|\left\langle\chi, B+\widehat{R} k^{2}, \Psi\right\rangle\right|+k^{3} \\
& =\left\|\left(P_{\mathcal{N}}^{\|} B+\widehat{R} k^{2}\right) \Psi\right\|+k^{3} \\
& \geq \inf _{\widetilde{\Psi} \in \mathcal{N},\|\widetilde{\Psi}\|=1}\left\|\left(P_{\mathcal{N}}^{\|} B+\widehat{R} k^{2}\right) \widetilde{\Psi}\right\|+k^{3} .
\end{aligned}
$$

Using this formula and $\bar{\lambda}=0$ in Lemma 5.5 the Corollary follows.

$\square$ For $\bar{\lambda}=1$ we have for Lemma 5.5

Corollary 5.7 Let $A \in \mathcal{C}$ and $\bar{\lambda}=1$ (i.e. $\mathcal{N} \cap L^{2}=\emptyset$ ). Then there exist constants $C, K, k_{0}>0$ such that for any normalized $\Phi \in \mathcal{N}$, any $\mathbf{k} \in \mathbb{R}^{3}$ with $k<k_{0}$ and any potential $B \in \mathcal{W}_{K}$

$$
\left\|P_{\mathcal{N}}^{\|}\left(1-T_{E_{k}}^{A+B}\right)^{-1}(A \Phi)\right\| \leq C\left(\inf _{\Psi \in \mathcal{N},\|\Psi\|=1}|\langle\Psi, B, \Psi\rangle|+k\right)^{-1}
$$

and

$$
\left\|P_{\mathcal{N}}^{\perp}\left(1-T_{E_{k}}^{A+B}\right)^{-1}(A \Phi)\right\|_{\infty} \leq C\left(k+\|B\|_{1}+\|B\|_{\infty}\right)\left(\inf _{\Psi \in \mathcal{N},\|\Psi\|=1}|\langle\Phi, B, \Phi\rangle|+k\right)^{-1}
$$

Furthermore we have for any normalized $m^{\perp} \in \mathcal{M}^{\perp}$

$$
\left\|P_{\mathcal{N}}^{\|}\left(1-T_{E_{k}}^{A+B}\right)^{-1} m^{\perp}\right\|_{\infty} \leq C k\left(\inf _{\Psi \in \mathcal{N},\|\Psi\|=1}|\langle\Phi, B, \Phi\rangle|+k\right)^{-1}
$$

and

$$
\left\|P_{\mathcal{N}}^{\perp}\left(1-T_{E_{k}}^{A+B}\right)^{-1} m^{\perp}\right\|_{\infty} \leq C .
$$

Proof: The proof is as the proof of Corollary[5.6]above, using that $B$ is selfadjoint and $q$ is anti-selfadjoint.

Next we show, how these corollaries imply the Theorem. First recall (18)

$$
\left(1-T_{E_{k}}^{A+B}\right) \phi(A+B, j, \mathbf{k}, \cdot)=\chi(j, \mathbf{k}, \cdot) .
$$


Defining

$$
g(A+B, j, \mathbf{k}, \cdot)=T_{E_{k}}^{A+B} \chi(j, \mathbf{k}, \cdot)
$$

and

$$
\zeta(A+B, j, \mathbf{k}, \cdot)=\phi(A+B, j, \mathbf{k}, \cdot)-\chi(j, \mathbf{k}, \cdot)
$$

it follows that

$$
\begin{aligned}
\zeta(A+B, j, \mathbf{k}, \cdot)= & -\left(1-T_{E_{k}}^{A+B}\right)^{-1} g(A+B, j, \mathbf{k}, \cdot) \\
= & -\left(1-T_{E_{k}}^{A+B}\right)^{-1} P_{\mathcal{M}}^{\|} g(A+B, j, \mathbf{k}, \cdot) \\
& -\left(1-T_{E_{k}}^{A+B}\right)^{-1} P_{\mathcal{M}}^{\perp} g(A+B, j, \mathbf{k}, \cdot)
\end{aligned}
$$

Proof of Theorem 3.4: Below we shall show that that for $\bar{\lambda}=0$

$$
\left\|P_{\mathcal{M}}^{\|} g(A+B, j, \mathbf{k}, \cdot)\right\|_{\infty}<C\left(k+\|B\|_{1}\right) .
$$

Defining

$$
\widetilde{\Phi}_{j, \mathbf{k}}^{B}:=P_{\mathcal{N}}^{\|} \zeta(A+B, j, \mathbf{k}, \cdot)
$$

and using Corollary 5.6 in (60) one gets

$$
\begin{aligned}
\left\|\widetilde{\Phi}_{j, \mathbf{k}}^{B}\right\| \leq & \left\|P_{\mathcal{N}}^{\|}\left(1-T_{E_{k}}^{A+B}\right)^{-1} P_{\mathcal{M}}^{\|} g(A+B, j, \mathbf{k}, \cdot)\right\|+\left\|P_{\mathcal{N}}^{\|}\left(1-T_{E_{k}}^{A+B}\right)^{-1} P_{\mathcal{M}}^{\perp} g(A+B, j, \mathbf{k}, \cdot)\right\| \\
\leq & C\left(k+\|B\|_{1}\right)\left(\inf _{\Psi \in \mathcal{N},\|\Psi\|=1}\left\|\left(P_{\mathcal{N}}^{\|} B+\widehat{R} k^{2}\right) \Psi\right\|+k^{3}\right)^{-1} \\
& +C k^{2}\left(\inf _{\Psi \in \mathcal{N},\|\Psi\|=1}\left\|\left(P_{\mathcal{N}}^{\|} B+\widehat{R} k^{2}\right) \Psi\right\|+k^{3}\right)^{-1} \\
\leq & C\left(k+\|B\|_{1}\right)\left(\inf _{\Psi \in \mathcal{N},\|\Psi\|=1}\left\|\left(P_{\mathcal{N}}^{\|} B+\widehat{R} k^{2}\right) \Psi\right\|+k^{3}\right)^{-1}
\end{aligned}
$$

and in view of (59)

$$
\begin{aligned}
& \left\|\phi(A+B, j, \mathbf{k}, \cdot)-\widetilde{\Phi}_{j, \mathbf{k}}^{B}\right\| \\
\leq & \|\chi(j, \mathbf{k}, \cdot)\|_{\infty}+\left\|P_{\mathcal{N}}^{\perp} \zeta(A+B, j, \mathbf{k}, \cdot)\right\|_{\infty} \\
\leq & 1+\left\|P_{\mathcal{N}}^{\perp}\left(1-T_{E_{k}}^{A+B}\right)^{-1} g(A+B, j, \mathbf{k}, \cdot)\right\|+\left\|P_{\mathcal{N}}^{\perp}\left(1-T_{E_{k}}^{A+B}\right)^{-1} P_{\mathcal{M}}^{\perp} g(A+B, j, \mathbf{k}, \cdot)\right\| \\
\leq & 1+C k\left(k^{2}+\|B\|_{1}+\|B\|_{\infty}\right)\left(\inf _{\Psi \in \mathcal{N},\|\Psi\|=1}\left\|\left(P_{\mathcal{N}}^{\|} B+\widehat{R} k^{2}\right) \Psi\right\|+k^{3}\right)^{-1}+C \\
\leq & C+C k\left(\|B\|_{1}+\|B\|_{\infty}\right)\left(\inf _{\Psi \in \mathcal{N},\|\Psi\|=1}\left\|\left(P_{\mathcal{N}}^{\|} B+\widehat{R} k^{2}\right) \Psi\right\|+k^{3}\right)^{-1} .
\end{aligned}
$$

These formulas imply the Theorem, it is left to verify (61). Using the equivalence of all norms in the finite dimensional space $\mathcal{M}$ we have that there exists a $C>0$ and a normalized $\Phi \in \mathcal{N}$ such that

$$
\left\|P_{\mathcal{M}}^{\|} g(A+B, j, \mathbf{k}, \cdot)\right\|_{\infty} \leq C\langle\Phi, A, g(A+B, j, \mathbf{k}, \cdot)\rangle
$$

In view of (58) we have

$$
\begin{aligned}
& |\langle\Phi, A, g(A+B, j, \mathbf{k}, \cdot)\rangle| \\
& =\left|\left\langle\Phi, A, T_{1}^{A+B} \chi(j, \mathbf{k}, \cdot)\right\rangle+\left\langle\Phi, A,\left(T_{E_{k}}^{A+B}-T_{1}^{A+B}\right) \chi(j, \mathbf{k}, \cdot)\right\rangle\right| \\
& \leq\left|\left\langle\Phi, A, T_{1}^{A+B} \chi(j, \mathbf{k}, \cdot)\right\rangle\right|+\left|\left\langle\left(T_{E_{k}}^{A}-T_{1}^{A}\right) \Phi, A+B, \chi(j, \mathbf{k}, \cdot)\right\rangle\right| \\
& \leq|\langle\Phi, A, g(A+B, j, 0, \cdot)\rangle|+\left|\left\langle\Phi, A, T_{1}^{A+B}\left(\chi(j, \mathbf{k}, \cdot)-\chi_{1}\right)\right\rangle\right| \\
& +\left\|\left(T_{E_{k}}^{A}-T_{1}^{A}\right) \Phi\right\|_{\infty}\|(A+B) \chi(j, \mathbf{k}, \cdot)\|_{1} .
\end{aligned}
$$


Remember, that $\chi(j, \mathbf{k}, \mathbf{c})=e^{i \mathbf{k} \cdot \mathbf{x}}$ multiplied with some $(k$-dependent) four-spinor. Hence $\chi(j, \mathbf{k}, x \cdot)-$ $\chi(j, 0, \mathbf{x})$ is of order $k(1+x)$, thus the second summand is of order $k$. In view of Lemma 6.5 (d) using that $\chi(j, \mathbf{k}, \cdot)$ is normalized, the third summand is of order $\left(\bar{\lambda} k+k^{2}\right)$. It suffices to prove that if $\bar{\lambda}=0$

$$
\langle\Phi, A, g(A+B, j, 0, \cdot)\rangle=\mathcal{O}\left(\|B\|_{1}\right) .
$$

Therefore we use that $\chi(j, 0, \cdot)$ is a generalized eigenfunction of the free Dirac equation with energy 1 , i.e. $(1-\beta) \chi(j, 0, \cdot)=0$ and thus $(1+\beta) \chi(j, 0, \cdot)=2 \chi(j, 0, \cdot)$. This (28), (58) and (15) yields

$$
\begin{aligned}
\langle\Phi, A, g(A+B, j, 0, \cdot)\rangle= & \left\langle\Phi, A, T_{1}^{A+B} \chi(j, 0, \cdot)\right\rangle \\
= & \frac{1}{2}\left\langle T_{1}^{A} \Phi, A+B,(1+\beta) \chi(j, 0, \cdot)\right\rangle \\
= & \frac{1}{2}\left\langle\int \Phi A(1+\beta) d^{3} x, A+B, \chi(j, 0, \cdot)\right\rangle \\
& +\frac{1}{2}\left\langle\int \Phi B(1+\beta) d^{3} x, A+B, \chi(j, 0, \cdot)\right\rangle \\
= & \frac{1}{2}\langle\lambda(\Phi), A+B, \chi(j, 0, \cdot)\rangle+\frac{1}{2}\left\langle\int \Phi B(1+\beta) d^{3} x, A+B, \chi(j, 0, \cdot)\right\rangle \\
\leq & C\|B\|_{1} .
\end{aligned}
$$

Theorem 3.5 follows with (59) and using Corollary 5.7 in (60).

\section{$6 \quad k$-Derivatives}

Next we will estimate the $k$-derivatives of the solutions of (18) assuming that $A$ and $B$ are compactly supported. The results of this section play an important role for the estimate of wave function decay (see [14] and [16]) via stationary phase method.

For ease of writing we define

$$
\alpha:=1+\left(k+\|B\|_{1}\right)\left(\inf _{\Phi \in \mathcal{N},\|\Phi\|=1}\left\|\left(P_{\mathcal{N}^{\|}}^{\|} B+\widehat{R} k^{2}\right) \Phi\right\|+k^{3}\right)^{-1}
$$

Heuristically deriving (18) with respect to $k$ yields $\partial_{k} \phi(A+B, j, \mathbf{k}, \mathbf{x})$. We denote the function we get by this formal method by $\dot{\phi}(A+B, j, \mathbf{k}, \mathbf{x})$.

$$
\left(1-T_{E_{k}}^{A+B}\right) \dot{\phi}(A+B, j, \mathbf{k}, \cdot)=\partial_{k} \chi_{\mathbf{k}}+\left(\partial_{k} T_{E_{k}}^{A+B}\right) \phi(A+B, j, \mathbf{k}, \cdot)=: f_{1} .
$$

Similarly as above one defines

$$
g^{1}:=\mu T_{E_{k}}^{A+B} f^{1} \text { and } \zeta^{1}:=\dot{\phi}-f^{1}
$$

to get

$$
\zeta^{1}(A+B, j, \mathbf{k}, \cdot)=-\left(1-T_{E_{k}}^{A+B}\right)^{-1} g^{1}(A+B, j, \mathbf{k}, \cdot) .
$$

In [5] it is shown that (66) has a unique solution and that in fact $\dot{\phi}=\partial_{k} \phi$.

Now $\dot{\phi}$ is controllable via $\zeta^{1}$ using (66) in a similar way as we controlled $\phi(A+B, j, \mathbf{k}, \cdot)$ above (c.f. (60) $)$. Let us heuristically estimate $\left\|\zeta^{1}(A+B, j, \mathbf{k}, \cdot)\right\|_{\infty}$ for $\bar{\lambda}=0$ to make the result clear, a rigorous treatment (which is in fact "not far" from this heuristics) shall be given below in more generality (i.e. for higher derivatives, also). Recall that $\|\phi(A+B, j, \mathbf{k}, \cdot)\|_{\infty} \leq C \alpha$ for appropriate $C<\infty$. Since

$$
\begin{aligned}
\left(\partial_{k} T_{E_{k}}^{A+B}\right) \phi(A+B, j, \mathbf{k}, \cdot) & =\left(\partial_{k} T_{E_{k}}^{A}\right) \phi(A+B, j, \mathbf{k}, \cdot)+\left(\partial_{k} T_{E_{k}}^{B}\right) \phi(A+B, j, \mathbf{k}, \cdot) \\
& =\left[\partial_{k} T_{E_{k}}^{A} \phi(A+B, j, \mathbf{k}, \cdot)\right]_{k=0}+\left(\mathcal{O}(k)+\mathcal{O}\left(\|B\|_{1}\right)\right)\|\phi(A+B, j, \mathbf{k}, \cdot)\|_{\infty}
\end{aligned}
$$

In view of (89) the first summand is zero for $\bar{\lambda}=0$ and $g^{1}$ is bounded from above by $C\left(k+\|B\|_{1}\right) \alpha$. Using as above Corollary [5.6] we get that $\partial_{k} \phi \leq C \alpha^{2}$ for appropriate $C$.

Heuristically one can treat the higher derivatives similarly, hence we have 
Theorem 6.1 Let $A \in \mathcal{C}$ with $\bar{\lambda}=0$ (i.e. $\mathcal{N} \subset L^{2}$ ). Then there exist constants $C, K, k_{0}>0$ and a selfadjoint linear map $\widehat{R}: \mathcal{N} \rightarrow \mathcal{N}$ such that for any $m \in \mathbb{N}_{0}$ there exist $C_{m}<\infty$ such that for any $\mathbf{k} \in \mathbb{R}^{3}$ with $k<k_{0}, j=1,2$, any potential $B \in \mathcal{W}_{K}$ there exists a $\Phi_{j, \mathbf{k}}^{B} \in \mathcal{N}$ with

$$
\left\|(1+x)^{-m} \partial_{k}^{m} \phi(A+B, j, \mathbf{k}, \cdot)\right\|_{\infty} \leq C_{m}\left(k^{-m}+\alpha^{m+1}\right) .
$$

Proof: We repeat the procedure above which gave us the defining equation for $\partial_{k} \phi$ (i.e. (65) for the higher derivatives. We get formally

$$
\partial_{k}^{m}\left(\left(1-T_{E_{k}}^{A+B}\right) \phi(A+B, j, \mathbf{k}, \cdot)\right)=\partial_{k}^{m} \chi(j, \mathbf{k}, \cdot),
$$

hence

$$
\left(\left(1-T_{E_{k}}^{A+B}\right) \phi^{(m)}(A+B, j, \mathbf{k}, \cdot)\right)=\partial_{k}^{m} \chi(j, \mathbf{k}, \cdot)-\sum_{l=1}^{m}\left(\begin{array}{c}
m \\
l
\end{array}\right) \partial_{k}^{l} T_{E_{k}}^{A+B} \partial_{k}^{(m-l)} \phi(A+B, j, \mathbf{k}, \cdot) .
$$

Defining

$$
\begin{gathered}
f^{m}(A+B, j, \mathbf{k}, \cdot):=\partial_{k}^{m} \chi(j, \mathbf{k}, \cdot)-\sum_{l=1}^{m}\left(\begin{array}{c}
m \\
l
\end{array}\right) \partial_{k}^{l} T_{E_{k}}^{A+B} \partial_{k}^{(m-l)} \phi(A+B, j, \mathbf{k}, \cdot), \\
g^{m}(A+B, j, \mathbf{k}, \cdot):=T_{E_{k}}^{A+B} f^{m}(A+B, j, \mathbf{k}, \cdot)
\end{gathered}
$$

and

$$
\zeta^{(m)}(A+B, j, \mathbf{k}, \cdot):=\phi^{(m)}(A+B, j, \mathbf{k}, \cdot)-f^{m}(A+B, j, \mathbf{k}, \cdot)
$$

it follows that

$$
\left(1-T_{E_{k}}^{A+B}\right) \zeta^{(m)}(A+B, j, \mathbf{k}, \cdot)=-g^{m}(A+B, j, \mathbf{k}, \cdot) .
$$

Again [5] shows that the formal differentiations yield the right functions, i.e. $\phi^{m}=\partial_{k}^{m} \phi$.

First we will show inductively that there exist $C_{m}<\infty$ and $\Phi_{m} \in \mathcal{N}$ such that

$$
\begin{aligned}
\left\|(1+x)^{-m+1} f^{m}(A+B, j, \mathbf{k}, \cdot)\right\|_{\infty} & \leq C_{m} \alpha\left(k^{-1}+\alpha\right)^{m-1} \\
\left\|\Phi_{m}\right\| & \leq C_{m} \alpha\left(k^{-1}+\alpha\right)^{m} \\
\left\|(1+x)^{-m+1}\left(\phi^{m}(A+B, j, \mathbf{k}, \cdot)-\Phi_{m}\right)\right\|_{\infty} & \leq C_{m} \alpha\left(k^{-1}+\alpha\right)^{m-1} .
\end{aligned}
$$

For $m=0$ these equations hold (remember that $f^{0}=\chi(j, \mathbf{k}, \cdot)$ ) due to Theorem 3.4 .

Next we show, that $M-1$ implies $M$. Assume, that (72)-(74) hold for all $m<M$. Let us verify first (72) for $M$. For (69) we can write

$$
\begin{aligned}
f^{M}(A+B, j, \mathbf{k}, \cdot):= & \partial_{k}^{M} \chi(j, \mathbf{k}, \cdot)-\sum_{l=2}^{M}\left(\begin{array}{c}
M \\
j
\end{array}\right) \partial_{k}^{l} T_{E_{k}}^{A+B} \partial_{k}^{(M-l)} \phi(A+B, j, \mathbf{k}, \cdot) \\
& +M \partial_{k} T_{E_{k}}^{A+B} \phi^{M-1}(A+B, j, \mathbf{k}, \cdot) .
\end{aligned}
$$

For compactly supported $A+B$ one has in view of (13) for any $\chi \in L^{\infty}$ that

$$
\begin{aligned}
\left\|(1+x)^{-M+1} \partial_{k}^{M} T_{E_{k}}^{A+B} \chi\right\|_{\infty} & \leq\|\chi\|_{\infty} \sup _{\mathbf{x} \in \mathbb{R}^{3}}\left|(1+x)^{-M+1} \int\right| \partial_{k}^{N} G_{E}^{+}(\mathbf{x}-\mathbf{y})|(A(\mathbf{y})+B(\mathbf{y}))| \\
& \leq C\left\|_{\chi}\right\|_{\infty} .
\end{aligned}
$$

Hence using (73) and (74) for $m<M$ it follows that

$$
\begin{aligned}
& \left\|(1+x)^{-M+1} f^{M}(A+B, j, \mathbf{k}, \cdot)\right\|_{\infty} \\
& =C+\sum_{l=2}^{M} C_{l} \alpha\left(k^{-1}+\alpha\right)^{M-l-1}+M\left\|\partial_{k} T_{E_{k}}^{A+B}\left(\phi^{M-1}(A+B, j, \mathbf{k}, \cdot)-\Phi_{M-1}\right)\right\|_{\infty} \\
& \quad+M\left\|\partial_{k} T_{E_{k}}^{A+B} \Phi_{M-1}\right\| . \\
& \leq C+C \alpha\left(k^{-1}+\alpha\right)^{M-l-1}+M\left\|\partial_{k} T_{E_{k}}^{A+B} \Phi_{M-1}\right\| .
\end{aligned}
$$


Below we will show, that $\left[\partial_{k} T_{E_{k}}^{A} \Phi\right]_{k=0}=\lambda(\Phi)$ (see (189) $)$, hence

$$
\begin{aligned}
\partial_{k} T_{E_{k}}^{A+B} \Phi & =\partial_{k} T_{E_{k}}^{A} \Phi+\partial_{k} T_{E_{k}}^{B} \Phi=\left[\partial_{k} T_{E_{k}}^{A} \Phi\right]_{k=0}+\mathcal{O}(k)+\mathcal{O}\left(\|B\|_{1}\right) \\
& =\mathcal{O}(k)+\mathcal{O}\left(\|B\|_{1}\right)
\end{aligned}
$$

for all $\Phi \in \mathcal{N}$. Hence

$$
\left\|(1+x)^{-M+1} f^{M}(A+B, j, \mathbf{k}, \cdot)\right\|_{\infty} \leq C+C \alpha\left(k^{-1}+\alpha\right)^{M-2}+C\left(k+\|B\|_{1}\right) \alpha\left(k^{-1}+\alpha\right)^{M-1} .
$$

Note that $\alpha>1$ and $\left(k^{-1}+\alpha\right)^{-1}<k$, hence

$$
\left\|(1+x)^{-M+1} f^{M}(A+B, j, \mathbf{k}, \cdot)\right\|_{\infty} \leq C\left(k+\|B\|_{1}\right) \alpha\left(k^{-1}+\alpha\right)^{M-1},
$$

which is (72) for $m=M$. It follows that also $\left\|g_{M+1}\right\|_{\infty} \leq C\left(k+\|B\|_{1}\right) \alpha\left(k^{-1}+\alpha\right)^{M-1}$. With Corollary 5.6 we get in view of (71) that

$$
\left\|\Phi_{m}\right\| \leq C_{m} \alpha^{2}\left(k^{-1}+\alpha\right)^{m-2} \leq C_{m} \alpha\left(k^{-1}+\alpha\right)^{m-1}
$$

and

$$
\left\|(1+x)^{-m+1}\left(\phi^{m}(A+B, j, \mathbf{k}, \cdot)-\Phi_{m}\right)\right\|_{\infty} \leq C_{m} \alpha\left(k^{-1}+\alpha\right)^{m-1} .
$$

which are (73) and (74) for $m=M$.

Induction over $m$ yields, that (72) - (74) hold for all $m \in \mathbb{N}_{0}$.

With (73) and (74) the Theorem follows easily. Since $\alpha>1$ we have that (i) if $\alpha>k^{-1}$ the right hand sides of (73) and (74) are bounded by $C_{m} 2^{m} \alpha^{m+1}$, (ii) if $\alpha \leq k^{-1}$ the right hand sides of (73) and (74) are bounded by $C_{m} 2^{m} \alpha k^{-m}$ and the Theorem follows.

Again we get in a similar but easier way the respective Theorem for $\bar{\lambda}=1$.

Theorem 6.2 Let $A \in \mathcal{C}$ with $\bar{\lambda}=1$. Then the respective statement of Theorem 6.1 holds with

$$
\alpha=1+\left(\inf _{\Phi \in \mathcal{N},\|\Phi\|=1}|\langle\Phi, B, \Phi\rangle|+k\right)^{-1}
$$

As above one can make it easier to understand the statement of Theorem 3.4, by restriction on potentials $B_{\mu}$ which can be written as $B_{\mu} \mu B_{0}$ for some fixed potential $B_{0}$ and $\mu \in\left[-\mu_{0}, \mu_{0}\right]$.

Corollary 6.3 Let $A \in \mathcal{C}$ with $\bar{\lambda}=0$. Let $B_{0} \in L^{\infty} \cap L^{1}$ with $\left\langle\Phi, B_{0}, \Phi\right\rangle \neq 0$ for all $\Phi \in \mathcal{N} \backslash\{0\}$. Then there exist constants $C, \mu_{0}, k_{0}>0$ and constants $\gamma_{l}, l=1, \ldots, m \leq \operatorname{dim} \mathcal{N}$ such that for any $m \in \mathbb{N}_{0}$ there exist $C_{m}<\infty$ such that for any $\mathbf{k} \in \mathbb{R}^{3}$ with $k<k_{0}, j=1,2$, any $\mu \in\left[-\mu_{0}, \mu_{0}\right]$ there exists a $\Phi_{j, \mathbf{k}}^{\mu} \in \mathcal{N}$ with

$$
\left\|(1+x)^{-m} \partial_{k}^{m} \phi\left(A+\mu B_{0}, j, \mathbf{k}, \cdot\right)\right\|_{\infty} \leq C_{m}\left(k^{-m}+\left|\sum_{l=1}^{n} \frac{k}{\left|\mu+\gamma_{l} k^{2}\right|+k^{3}}\right|^{m+1}\right)
$$

This Corollary can now be used to give estimates on the behavior of the generalized eigenfunctions for critical potentials multiplied with a factor close to one (i.e. considering the case $A+B=\lambda \mu A$ with $\mu \approx 1$ ). Such potentials are a comparably easy model to estimate physical processes under the influence of critical fields with small perturbations. Therefore the literature on Adiabatic Pair Creation (see e.g. [12, 13]) deals with potentials $A$ multiplied by a switching factor. We shall give a result suitable for such application, imposing further conditions on the potential $A$ which allow us to extend the bounds on $\mathbf{k} \in \mathbb{R}^{3}$. The following Corollary shall play an important role in the proof of adiabatic pair creation which has been achieved recently [16] 
Corollary 6.4 Let $A \in \mathcal{C}$ be positive and purely electric with $\bar{\lambda}=0$. Then there exist constants $C, \delta>0$ and constants $\gamma_{l}, l=1, \ldots, m \leq \operatorname{dim} \mathcal{N}$ such that for any $m \in \mathbb{N}_{0}$ there exist $C_{m}<\infty$ such that for any $\mathbf{k} \in \mathbb{R}^{3}, j=1,2$, any $\mu \in[1-\delta, 1+\delta]$ there exists a $\Phi_{j, \mathbf{k}}^{\mu} \in \mathcal{N}$ with

$$
\left\|(1+x)^{-m} \partial_{k}^{m} \phi(\mu A, j, \mathbf{k}, \cdot)\right\|_{\infty} \leq C_{m}\left(k^{-m}+\left|\sum_{l=1}^{n} \frac{k}{\left|\mu+\gamma_{l} k^{2}\right|+k^{3}}\right|^{m+1}\right) .
$$

Furthermore there exist $\Phi_{\mu}(\mathbf{k}, j, \cdot) \in \mathcal{N}$ and $C$ uniform in $\mathbf{k} \in \mathbb{R}^{3}$ and $\mu \in[1-\delta, 1+\delta]$ so that

$$
\left\|\phi(\mu A, j, \mathbf{k}, \cdot)-\Phi_{\mu}(\mathbf{k}, j, \cdot)\right\|_{\infty}<C .
$$

Proof: For $k$ smaller than $k_{0}$ the Corollary follows from Corollary 6.3 and Corollary 3.7 replacing $\mu$ by $1-\mu$ and setting $B_{0}=A$. Note, that for positive $A$ one has $\langle\Phi, A, \Phi\rangle>0$ for all $\Phi \in \mathcal{N}$, hence the assumptions on $B_{0}$ in Lemma 6.3 are satisfied for $A$.

Using continuity of the operator $T$ one can find a uniform bound on $\|\phi(\mu A, j, \mathbf{k}, \cdot)\|_{\infty}$ for $\mathbf{k}$ in an arbitrary compact subset of $\mathbb{R}^{3}$ not containing $k=0$ (see for example [5]). In [5] it is also proven that the left hand side of (76) is bounded for $k \rightarrow \infty$ and the Corollary follows.

\section{Acknowledgments}

The author would like to thank Detlef Dürr for suggesting to work on the topic. The discussions with him were very helpful and influenced the paper a lot.

Financial support by the Austrian Science Fund in the form of an Erwin Schrödinger Fellowship is gratefully acknowledged.

\section{Appendix}

\section{Control of $\Phi_{1}$ in (14)}

Above we showed, that any element $\Phi \in \mathcal{N}$ is in $L^{2}$ if and only if $\lambda(\Phi)=0$. There we split $\Phi=$ $\Phi_{1}+x^{-1} \lambda(\Phi)$. Assuming that $\Phi_{1}(\mathbf{x})$ decays at least as fast as $x^{-2}$ and using $\Phi \in \mathcal{B} \subset L^{\infty}$ it in fact follows that $\Phi \in L^{2} \Leftrightarrow \lambda(\Phi)=0$.

Let us now proof that under our assumptions of the potential we always have that $\Phi_{1}(\mathbf{x})$ decays at least as fast as $x^{-2}$. 
For $\Phi_{1}(\mathbf{x})($ c.f (14) $)$ we can write defining $f(\mathbf{x}):=(1+x)^{2} A(\mathbf{x}) \Phi(\mathbf{x})$

$$
\begin{aligned}
\left|\Phi_{1}(\mathbf{x})\right| \leq & \left.\mid \int_{y<\frac{x}{2}} \frac{1}{4 \pi}\left(\frac{y-x}{y x}(1+\beta)+i \sum_{j=1}^{3} \alpha_{j} \frac{y_{j}}{y^{3}}\right)\right) A(\mathbf{x}-\mathbf{y}) \Phi(\mathbf{x}-\mathbf{y}) d^{3} y \mid \\
& \left.+\mid \int_{y \geq \frac{x}{2}} \frac{1}{4 \pi}\left(\frac{y-x}{y x}(1+\beta)+i \sum_{j=1}^{3} \alpha_{j} \frac{y_{j}}{y^{3}}\right)\right) A(\mathbf{x}-\mathbf{y}) \Phi(\mathbf{x}-\mathbf{y}) d^{3} y \mid \\
\leq & \left.\mid \int_{y<\frac{x}{2}} \frac{1}{4 \pi}(1+|\mathbf{x}-\mathbf{y}|)^{-2}\left(\frac{y-x}{y x}(1+\beta)+i \sum_{j=1}^{3} \alpha_{j} \frac{y_{j}}{y^{3}}\right)\right) f(\mathbf{x}-\mathbf{y}) d^{3} y \mid \\
& \left.+\mid \int_{y \geq \frac{x}{2}} \frac{1}{4 \pi}\left(\frac{1}{y x}(1+\beta)+i \sum_{j=1}^{3} \alpha_{j} \frac{y_{j}}{y^{3}}\right)\right) f(\mathbf{x}-\mathbf{y}) d^{3} y \mid \\
\leq & \left.\left(1+\frac{x}{2}\right)^{-2} \mid \int_{y \leq 1} \frac{1}{4 \pi}\left(\frac{y-x}{y x}(1+\beta)+i \sum_{j=1}^{3} \alpha_{j} \frac{y_{j}}{y^{3}}\right)\right) f(\mathbf{x}-\mathbf{y}) d^{3} y \mid \\
& \left.+\left(1+\frac{x}{2}\right)^{-2} \mid \int_{1<y<\frac{x}{2}} \frac{1}{4 \pi}\left(\frac{y-x}{y x}(1+\beta)+i \sum_{j=1}^{3} \alpha_{j} \frac{y_{j}}{y^{3}}\right)\right) f(\mathbf{x}-\mathbf{y}) d^{3} y \mid \\
& +\frac{2}{x^{2}}\left|\int_{y \geq \frac{x}{2}} \frac{1}{4 \pi} f(\mathbf{x}-\mathbf{y}) d^{3} y\right| \\
\leq & \left.\left(1+\frac{x}{2}\right)^{-2}\|f\|_{\infty} \mid \int_{y \leq 1} \frac{1}{4 \pi}\left(\frac{y-x}{y x}(1+\beta)+i \sum_{j=1}^{3} \alpha_{j} \frac{y_{j}}{y^{3}}\right)\right) d^{3} y \mid \\
& +\left(1+\frac{x}{2}\right)^{-2}\left|\int_{1<y<\frac{x}{2}} \frac{1}{4 \pi}\left(3+\frac{2}{x}\right) f(\mathbf{x}-\mathbf{y}) d^{3} y\right|+\frac{2}{x^{2}}\left|\int_{y \geq \frac{x}{2}} \frac{1}{4 \pi} f(\mathbf{x}-\mathbf{y}) d^{3} y\right|
\end{aligned}
$$

Since $(1+x)^{2} A \in L^{1} \cap L^{\infty}$ and $\Phi \in \mathcal{B} \subset L^{\infty}$ we have that $f \in L^{1} \cap L^{\infty}$. Thus $\Phi_{1}(\mathbf{x})$ decays like $x^{-2}$, it follows that $\Phi_{1} \in L^{2}$.

\section{Proof of Lemma 5.4}

Next we shall prove the following Lemma, the last points of which are exactly Lemma 5.4 .

Lemma 6.5 Let $A \in \mathcal{C}$. Then there exist constants $C, C^{\prime}>0, C_{0}, C_{1} \in \mathbb{R}$, a selfadjoint sesquilinear map $r: \mathcal{N} \times \mathcal{N} \rightarrow \mathbb{C}$ and an anti-selfadjoint sesquilinear map $s: \mathcal{N} \times \mathcal{N} \rightarrow \mathbb{C}$ with $s(\chi, \chi) \neq 0$ for all $\chi \in \mathcal{N}$ such that for any $\mathbf{k} \in \mathbb{R}^{3}$ with $k<1$, any potential $B$ with $B \in L^{1} \cap L^{\infty}$ and any normalized $h \in L^{\infty}$, normalized $m^{\perp} \in \mathcal{M}^{\perp}$ and normalized $\Phi, \Psi \in \mathcal{N}$

(a)

(b)

$$
\left\|\left(T_{E_{k}}^{A}-1\right) m^{\perp}\right\|_{\infty} \geq C
$$

$$
\left\|\left(T_{E_{k}}^{A}-T_{1}^{A}\right) h\right\|_{\infty}<C k
$$

(c)

$$
\left\|P_{\mathcal{M}}^{\perp} h\right\|_{\infty} \leq C,
$$

(d)

$$
\left\|\left(T_{E_{k}}^{A}-T_{1}^{A}\right) \Phi\right\|_{\infty} \leq C\left(\bar{\lambda} k+k^{2}\right)
$$


(e)

(f)

$$
\left|\left\langle\Phi, A,\left(T_{E_{k}}^{A+B}-T_{1}^{A+B}\right) h\right\rangle\right|<C\left(\|A\|_{1}+\|B\|_{1}\right)\left(\bar{\lambda} k+k^{2}\right),
$$

(g)

$$
\left|\left\langle\Phi, A,\left(1-T_{E_{k}}^{A+B}\right) m^{\perp}\right\rangle\right|<C\left(\|A\|_{1}+\|B\|_{1}\right)\left(\bar{\lambda} k+k^{2}\right),
$$

$$
\left\|P_{\mathcal{M}}^{\perp}\left(1-T_{E_{k}}^{A+B}\right) m^{\perp}\right\|_{\infty} \geq C-C^{\prime}\left(\bar{\lambda} k+k^{2}+\|B\|_{1}+\|B\|_{\infty}\right)
$$

(h)

$$
\left\|P_{\mathcal{M}}^{\perp}\left(1-T_{E_{k}}^{A+B}\right) \Phi\right\|_{\infty}<C\left(\bar{\lambda} k+k^{2}+\|B\|_{1}+\|B\|_{\infty}\right),
$$

(i)

$$
\left\langle\Phi, A,\left(T_{1}^{B}-T_{E_{k}}^{B}\right) \Psi\right\rangle \leq\|B\|_{1}\left(\bar{\lambda} \mathcal{O}(k)+\mathcal{O}\left(k^{2}\right)\right)
$$

and if $B=A$

$$
\left\langle\Phi, A,\left(T_{1}^{A}-T_{E_{k}}^{A}\right) \Psi\right\rangle=-i k\langle\Phi, A, \lambda(\Psi)\rangle+r(\Phi, \Psi) k^{2}+s(\Phi, \Psi) k^{3}+o\left(k^{3}\right),
$$

(j)

$$
\begin{aligned}
\left\langle\Phi, A,\left(1-T_{E_{k}}^{A+B}\right) \Psi\right\rangle= & \langle\Phi, B, \Psi\rangle-i k\langle\Phi, A, \lambda(\Psi)\rangle+r(\Phi, \Psi) k^{2}+s(\Phi, \Psi) k^{3} \\
& +o\left(k^{3}\right)+\|B\|_{1}\left(\bar{\lambda} \mathcal{O}(k)+\mathcal{O}\left(k^{2}\right)\right)
\end{aligned}
$$

Proof of (a) Let $k \in \mathbb{R}, m^{\perp} \in \mathcal{M}^{\perp}$. We will prove part (a) of the Lemma by contradiction. Assume that for every $n \in \mathbb{N}$ there exists a $k_{0} \leq k_{n}<1$ and a function $h_{n} \in \mathcal{M}^{\perp}$ with $\left\|h_{n}\right\|_{\infty}=1$ such that

$$
\left\|\left(1-T_{E_{k_{n}}}^{A}\right) h_{n}\right\|_{\infty}<\frac{1}{n}
$$

i.e.

$$
\lim _{n \rightarrow \infty}\left(1-T_{E_{k_{n}}}^{A}\right) h_{n}=0
$$

Using Bolzano Weierstraß we can assume without loss of generality that $k_{n}$ converges. We denote the respective limit by $k_{0}$. Using that $T_{E_{k}}^{A}$ is completely continuous it follows that

$$
\lim _{n \rightarrow \infty}\left(1-T_{E_{k_{0}}}^{A}\right) h_{n}=0 .
$$

But the sequence $T_{E_{k_{0}}}^{A} h_{n}$ is Arzela-Ascoli compact, since

$$
\mathcal{A}:=\left\{T_{E_{k_{0}}}^{A} g \text { with } g \in \mathcal{B},\|g\|_{\infty}=1\right\}
$$

is compact in the Arzela-Ascoli sense, i.e. for any $\varepsilon>0$ there exists a $\delta>0$ such that

$$
|f(\mathbf{x})-f(\mathbf{y})|<\varepsilon
$$

for all $\mathbf{x}, \mathbf{y} \in \mathbb{R}^{3}$ with $\|\mathbf{x}-\mathbf{y}\|<\delta$ and all $f \in \mathcal{A}$.

To prove this let $\varepsilon>0, f \in \mathcal{A}$ and let $k \in \mathbb{R}$ and $g \in \mathcal{B}$ be such that $f=T_{E_{k_{0}}}^{A} g,\|g\|_{\infty}=1$.

Then

$$
\begin{aligned}
|f(\mathbf{x})-f(\mathbf{y})| & =\left|T_{E_{k_{0}}}^{A} g(\mathbf{x})-T_{E_{k_{0}}}^{A} g(\mathbf{y})\right| \\
& =\left|\int G_{E_{k_{0}}}^{+}(\mathbf{x}-\mathbf{z}) A(\mathbf{z}) g(\mathbf{z}) d^{3} z-\int G_{E_{k_{0}}}^{+}(\mathbf{y}-\mathbf{z}) A(\mathbf{z}) g(\mathbf{z}) d^{3} z\right| \\
& =\left|\int\left(G_{E_{k_{0}}}^{+}(\mathbf{x}-\mathbf{z})-G_{E_{k_{0}}}^{+}(\mathbf{y}-\mathbf{z})\right) A(\mathbf{z}) g(\mathbf{z}) d^{3} z\right| .
\end{aligned}
$$


For any $\zeta>0$ we can write

$$
\begin{aligned}
|f(\mathbf{x})-f(\mathbf{y})| \leq & \left|\int_{z<\zeta}\left(G_{E_{k_{0}}}^{+}(\mathbf{x}-\mathbf{z})-G_{E_{k_{0}}}^{+}(\mathbf{y}-\mathbf{z})\right) A(\mathbf{z}) g(\mathbf{z}) d^{3} z\right| \\
& +\left|\int_{z>\zeta}\left(G_{E_{k_{0}}}^{+}(\mathbf{x}-\mathbf{z})-G_{E_{k_{0}}}^{+}(\mathbf{y}-\mathbf{z})\right) A(\mathbf{z}) g(\mathbf{z}) d^{3} z\right| \\
\leq & \|A(\mathbf{z})\|_{\infty}\|g(\mathbf{z})\|_{\infty}\left|\int_{z<\zeta}\left(G_{E_{k_{0}}}^{+}(\mathbf{x}-\mathbf{z})-G_{E_{k_{0}}}^{+}(\mathbf{y}-\mathbf{z})\right) d^{3} z\right| \\
& +\sup _{r>\zeta} G_{E_{k_{0}}}^{+}(\mathbf{x}-\mathbf{r})-G_{E_{k_{0}}}^{+}(\mathbf{y}-\mathbf{r})\|A(\mathbf{z})\|_{1}\left\|_{1}\right\|_{\infty} .
\end{aligned}
$$

Since $G_{E_{k_{0}}}^{+}$is integrable, the first summand goes to zero in the limit $\zeta \rightarrow 0$. Hence we can find a $\zeta>0$ such that the first summand is smaller than $\varepsilon / 2$.

Since $G_{E_{k_{0}}}^{+}$is on any set bounded away from 0 uniformly continuous, the second summand goes for any fixed $\zeta>0$ to zero in the limit $|\mathbf{x}-\mathbf{y}| \rightarrow 0$. Hence we can find for any $\zeta>0$ a $\delta>0$ such that the second summand is smaller than $\varepsilon / 2$. It follows that $|f(\mathbf{x})-f(\mathbf{y})|<\varepsilon$ for $\|\mathbf{x}-\mathbf{y}\|<\delta$.

It follows that $\mathcal{A}$ is compact (in the Arzela-Ascoli sense).

Thus there exists a convergent subsequence

$$
\left(T_{E_{k_{n(j)}}}^{A} h_{n(j)}\right)_{j \in \mathbb{N}}
$$

of $\left(T_{E_{k_{0}}}^{A} h_{n}\right)_{n \in \mathbb{N}}$ with $\lim _{j \rightarrow \infty} T_{E_{k_{n(j)}}}^{A} h_{n(j)}=h \in \mathcal{A}$ (i.e. $\|h\|_{\infty}=1$ ).

By virtue of (81) $\lim _{j \rightarrow \infty} h_{n(j)}=\lim _{j \rightarrow \infty} T_{E_{k_{0}}}^{A} h_{n(j)}=h$ and $\left(1-T_{E_{k_{0}}}^{A}\right) h=0$. Since $\left(1-T_{E_{k_{0}}}^{A}\right) h=0$ has nontrivial solutions only for $k_{0}=0$ it follows that $k_{0}=0$ and $h \in \mathcal{N}$.

On the other hand since $h_{n} \in \mathcal{M}^{\perp}$

$$
\left\langle\mathcal{N}, A, h_{n}\right\rangle=0
$$

for all $n \in \mathbb{N}$. With the continuity of the scalar product it follows that $h \in \mathcal{M}^{\perp}$, which contradicts to the fact that $\mathcal{N} \cap \mathcal{M}^{\perp}=\{0\}$ and part a) of the Lemma follows.

Proof of (b) Let $h \in L^{\infty}, A \in \mathcal{C}$. We have using (8)

$$
\left(T_{E_{k}}^{A}-T_{1}^{A}\right) h=\int\left(G_{E_{k}}^{+}(\mathbf{y})-G_{1}^{+}(\mathbf{y})\right) A(\mathbf{x}-\mathbf{y}) h(\mathbf{x}-\mathbf{y}) d^{3} y .
$$

It follows that

$$
\begin{aligned}
\left\|\left(T_{E_{k}}^{A}-T_{1}^{A}\right) h\right\|_{\infty} & \leq\|h\|_{\infty} \int\left|G_{E_{k}}^{+}(\mathbf{y})-G_{1}^{+}(\mathbf{y})\right| A(\mathbf{x}-\mathbf{y}) d^{3} y \\
& \leq\|h\|_{\infty}\left\|\frac{|\cdot|+1}{|\cdot|} A\right\|_{1}\left\|\frac{|\cdot|}{|\cdot|+1}\left(G_{E_{k}}^{+}(\cdot)-G_{1}^{+}(\cdot)\right)\right\|_{\infty} .
\end{aligned}
$$

Note that since $A \in L^{1} \cap L^{\infty}$ the $\left\|\frac{|\cdot|+1}{|\cdot|} A\right\|_{1}$ exists. 
Using the definition of $G_{E_{k}}^{+}($see (13) $)$we have that

$$
\begin{aligned}
\left\|\frac{|\cdot|}{|\cdot|+1}\left(G_{E_{k}}^{+}(\cdot)-G_{1}^{+}(\cdot)\right)\right\|_{\infty} \\
\quad=\| \frac{1}{4 \pi} \frac{e^{i k x}}{x+1}\left(-\left(E_{k}+\sum_{j=1}^{3} \alpha_{j} k \frac{x_{j}}{x}+\beta\right)-i x^{-1} \sum_{j=1}^{3} \alpha_{j} \frac{x_{j}}{x}\right) \\
\quad-\frac{1}{4 \pi} \frac{1}{x+1}\left(-(1+\beta)-i x^{-1} \sum_{j=1}^{3} \alpha_{j} \frac{x_{j}}{x}\right) \|_{\infty} \\
\leq\left\|\frac{1}{4 \pi} \frac{e^{i k x}}{x+1}\left(-\left(E_{k}-1+\sum_{j=1}^{3} \alpha_{j} k \frac{x_{j}}{x}\right)\right)\right\|_{\infty} \\
\quad+\left\|\frac{1}{4 \pi} \frac{e^{i k x}-1}{x+1}\left(-(1+\beta)-i x^{-2} \sum_{j=1}^{3} \alpha_{j} \frac{x_{j}}{x}\right)\right\|_{\infty} .
\end{aligned}
$$

The first summand is of order $k$. Since $e^{i k x}-1$ is of order $k x$, the second summand is of order $k$ and part (b) of the Lemma follows.

Proof of (c) The triangle inequality yields

$$
\left\|P_{\mathcal{M}}^{\perp} h\right\|_{\infty} \leq\left\|P_{\mathcal{M}}^{\|} h\right\|_{\infty}+\|h\|_{\infty}
$$

Since $\mathcal{M}^{\|}$has finite dimension, all norms on this space are equivalent, i.e. there exists a $C<0$ such that

$$
\begin{aligned}
\left\|P_{\mathcal{M}}^{\|} h\right\|_{\infty} & \leq C\left\|P_{\mathcal{M}}^{\|} h\right\|=: C \sup _{\Phi \in \mathcal{N}}\|A \Phi\|^{-1} \int A(\mathbf{x}) \Phi(\mathbf{x}) h(\mathbf{x}) d^{3} x \\
& \leq C\|h\|_{\infty}\|A\|_{1} \sup _{\Phi \in \mathcal{N}}\|A \Phi\|^{-1}\|\Phi\|_{\infty} .
\end{aligned}
$$

Using the equivalence of all norms on the finitely dimensional vector-space $\mathcal{M}^{\|}$we have that $\|A \Phi\|^{-1}\|\Phi\|_{\infty}$ is bounded and part (c) of the Lemma follows.

Proof of (i) Let $\Phi \in \mathcal{N}$ with $\|\Phi\|_{\infty}=1$. Using linearity it suffices to prove equation (78) for $B$ with $\|B\|_{1}=1$.

We shall use Taylors formula to estimate $\left\langle\Phi, B,\left(T_{1}^{A}-T_{E_{k}}^{a}\right) \Psi\right\rangle$. In view of (8)

$$
T_{E_{k}}^{A} \Phi=\int G_{E_{k}}^{+}(\mathbf{y}) A(\mathbf{x}-\mathbf{y}) \Phi(\mathbf{x}-\mathbf{y}) d^{3} y,
$$

i.e. we develop $G_{E_{k}}^{+}$(see (13) $)$around $k=0$, so we need the following derivatives

$$
\begin{aligned}
\partial_{k} G_{E_{k}}^{+} & =\partial_{k}\left(\frac{1}{4 \pi} e^{i k x}\left(-x^{-1}\left(E_{k}+\sum_{j=1}^{3} \alpha_{j} k \frac{x_{j}}{x}+\beta\right)-i x^{-2} \sum_{j=1}^{3} \alpha_{j} \frac{x_{j}}{x}\right)\right) \\
& =\frac{e^{i k x}}{4 \pi}\left(-i\left(E_{k}+\sum_{j=1}^{3} \alpha_{j} k \frac{x_{j}}{x}+\beta\right)+x^{-1} \sum_{j=1}^{3} \alpha_{j} \frac{x_{j}}{x}-x^{-1}\left(\frac{k}{E_{k}}+\sum_{j=1}^{3} \alpha_{j} \frac{x_{j}}{x}\right)\right) \\
& =\frac{e^{i k x}}{4 \pi}\left(-i\left(E_{k}+\sum_{j=1}^{3} \alpha_{j} k \frac{x_{j}}{x}+\beta\right)-x^{-1} \frac{k}{E_{k}}\right),
\end{aligned}
$$




$$
\begin{aligned}
\partial_{k}^{2} G_{E_{k}}^{+} & =\partial_{k}\left(\frac{1}{4 \pi} e^{i k x}\left(-i\left(E_{k}+\sum_{j=1}^{3} \alpha_{j} k \frac{x_{j}}{x}+\beta\right)-x^{-1} \frac{k}{E_{k}}\right)\right) \\
& =\frac{e^{i k x}}{4 \pi}\left(x\left(E_{k}+\sum_{j=1}^{3} \alpha_{j} k \frac{x_{j}}{x}+\beta\right)-i \frac{k}{E_{k}}-i \frac{k}{E_{k}}-i \sum_{j=1}^{3} \alpha_{j} \frac{x_{j}}{x}-x^{-1} \frac{1}{E_{k}^{3}}\right) \\
& =\frac{e^{i k x}}{4 \pi}\left(x\left(E_{k}+\sum_{j=1}^{3} \alpha_{j} k \frac{x_{j}}{x}+\beta\right)-2 i \frac{k}{E_{k}}-i \sum_{j=1}^{3} \alpha_{j} \frac{x_{j}}{x}-x^{-1} \frac{1}{E_{k}^{3}}\right)
\end{aligned}
$$

and

$$
\begin{aligned}
\partial_{k}^{3} G_{E_{k}}^{+}= & \partial_{k}\left(\frac{1}{4 \pi} e^{i k x}\left(x\left(E_{k}+\sum_{j=1}^{3} \alpha_{j} k \frac{x_{j}}{x}+\beta\right)-2 i \frac{k}{E_{k}}-i \sum_{j=1}^{3} \alpha_{j} \frac{x_{j}}{x}-x^{-1} \frac{1}{E_{k}^{3}}\right)\right) \\
= & \frac{e^{i k x}}{4 \pi}\left(i x^{2}\left(E_{k}+\sum_{j=1}^{3} \alpha_{j} k \frac{x_{j}}{x}+\beta\right)+2 x \frac{k}{E_{k}}+\sum_{j=1}^{3} \alpha_{j} x_{j}-i \frac{1}{E_{k}^{3}}\right. \\
& \left.+x \frac{k}{E_{k}}+\sum_{j=1}^{3} \alpha_{j} x_{j}-2 i \frac{1}{E_{k}^{3}}+3 x^{-1} \frac{k}{E_{k}^{5}}\right) \\
= & \frac{e^{i k x}}{4 \pi}\left(i x^{2}\left(E_{k}+\sum_{j=1}^{3} \alpha_{j} k \frac{x_{j}}{x}+\beta\right)+3 x \frac{k}{E_{k}}\right. \\
& \left.+2 \sum_{j=1}^{3} \alpha_{j} x_{j}-3 i \frac{1}{E_{k}^{3}}+3 x^{-1} \frac{k}{E_{k}^{5}}\right) .
\end{aligned}
$$

By Taylors formula we have that

$$
\begin{aligned}
\left\langle\Phi, B, T_{E_{k}}^{A} \Psi\right\rangle= & k\left[\partial_{k}\left\langle\Phi, B, T_{E_{k}}^{A} \Psi\right\rangle\right]_{k=0}+\frac{1}{2} k^{2}\left[\partial_{k}^{2}\left\langle\Phi, B, T_{E_{k}}^{A} \Psi\right\rangle\right]_{k=0}+o\left(k^{2}\right) \\
=: & S_{1}+S_{2}+\mathrm{o}\left(k^{2}\right) .
\end{aligned}
$$

For $S_{1}$ we obtain with (85) that

$$
\left[\partial_{k} T_{E_{k}}^{A}\right]_{k=0} \Psi=-i \int \frac{1}{4 \pi}(1+\beta) A(\mathbf{x}-\mathbf{y}) \Psi(\mathbf{x}-\mathbf{y}) d^{3} y=\lambda(\Psi) .
$$

Hence by (15)

$$
S_{1}=-1 k\langle\Phi, B, \lambda(\Psi)\rangle .
$$

For $S_{2}$ we have

$$
S_{2}=\frac{1}{2} k^{2}\left\langle\Psi, B, \int\left[\partial_{k}^{2} G_{E_{k}}^{+}(\mathbf{x}-\mathbf{y})\right]_{k=0} A(\mathbf{y}) \Phi(\mathbf{y}) d^{3} y\right\rangle .
$$

In view of (86) we have that for any $k_{0}>0$ there exists a $C>0$ such that

$$
\left|\left[\partial_{k}^{2} G_{E_{k}}^{+}(\mathbf{x}-\mathbf{y})\right]_{k=0}\right| \leq C\left(|\mathbf{x}-\mathbf{y}|+|\mathbf{x}-\mathbf{y}|^{-1}\right)
$$

uniform in $k<k_{0}$. Hence

$$
\begin{aligned}
\left|\int \partial_{k}^{2} G_{E_{k}}^{+}(\mathbf{x}-\mathbf{y}) A(\mathbf{y}) \Phi(\mathbf{y}) d^{3} y\right| \leq & \int_{|\mathbf{x}-\mathbf{y}|<1} 2 C|\mathbf{x}-\mathbf{y}|^{-1}|A(\mathbf{y}) \Phi(\mathbf{y})| d^{3} y \\
& +\int_{|\mathbf{x}-\mathbf{y}|>1} 2 C|\mathbf{x}-\mathbf{y} \| A(\mathbf{y}) \Phi(\mathbf{y})| d^{3} y \\
\leq & C\|A\|_{\infty}\|\Phi\|_{\infty}+C \int_{|\mathbf{x}-\mathbf{y}|>1}(x+y)|A(\mathbf{y}) \Phi(\mathbf{y})| d^{3} y .
\end{aligned}
$$


Since $(1+|\cdot|) A \in L^{1}$ and $\Phi \in L^{\infty}$ it follows that there exists a $C>0$ such that

$$
\int \partial_{k}^{2} G_{E_{k}}^{+}(\mathbf{x}-\mathbf{y}) A(\mathbf{y}) \Phi(\mathbf{y}) d^{3} y \leq C(1+x) .
$$

Hence

$$
\left|S_{2}\right| \leq \frac{1}{2} k^{2} C\langle\Psi, B,(1+x)\rangle .
$$

Using that $B \in L^{1}$ and that $(1+x) \Psi \in L^{\infty}$ (see below (15)) (78) follows.

Next we prove (79). We have by Taylors formula that

$$
\begin{aligned}
\left\langle\Phi, A, T_{E_{k}}^{A} \Psi\right\rangle= & k\left[\partial_{k}\left\langle\Phi, A, T_{E_{k}}^{A} \Psi\right\rangle\right]_{k=0} \\
+ & +\frac{1}{2} k^{2}\left[\partial_{k}^{2}\left\langle\Phi, A, T_{E_{k}}^{A} \Psi\right\rangle\right]_{k=0} \\
& +\frac{1}{6} k^{3}\left[\partial_{k}^{3}\left\langle\Phi, A, T_{E_{k}}^{A} \Psi\right\rangle\right]_{k=0} \\
& +\mathrm{o}\left(k^{3}\right) \\
=: & k \widetilde{q}(\Phi, \Psi)+k^{2} r(\Phi, \Psi)+k^{3} s(\Phi, \Psi)+\mathrm{o}\left(k^{3}\right) .
\end{aligned}
$$

Setting $B=A$ in the estimates above (see (90) and below) we get that there exists a $C_{1} \in \mathbb{R}$ such that $\widetilde{q}(\Phi, \Psi)=-i k\langle\Phi, A, \lambda(\Psi)\rangle$ and that $k^{2} r$ is well defined. Similarly we can show that $s$ is well defined, now using that $\left(1+x^{2}\right) A \in L^{1}$.

Using the symmetry of the operator $T_{E_{k}}^{A}$ and the symmetry of $i \partial_{k}$ we have that $r$ is selfadjoint and $s$ is anti-selfadjoint.

It is left to show, that $s(\chi, \chi) \neq 0$ for all $\chi \in \mathcal{N}$. Let $\chi \in \mathcal{N}$. We obtain by (87)

$$
\begin{aligned}
s(\chi, \chi)= & -\frac{1}{6}\left\langle\int \partial_{k}^{3} G_{E_{k}}^{+}(\mathbf{x}-\mathbf{y}) A(\mathbf{y}) \chi(\mathbf{y}) d^{3} y, A, \chi\right\rangle \\
= & -\frac{1}{6}\left\langle\frac{1}{4 \pi} \int i(\mathbf{x}-\mathbf{y})^{2}(1+\beta) A(\mathbf{y}) \chi(\mathbf{y}) d^{3} y, A, \chi\right\rangle \\
& -\frac{1}{6}\left\langle\frac{1}{4 \pi} \int 2 \sum_{j=1}^{3} \alpha_{j}\left(x_{j}-y_{j}\right) A(\mathbf{y}) \chi(\mathbf{y}) d^{3} y, A, \chi\right\rangle \\
& -\frac{1}{6}\left\langle\frac{1}{4 \pi} \int 3 i \frac{1}{m^{3}} A(\mathbf{y}) \chi(\mathbf{y}) d^{3} y, A, \chi\right\rangle \\
= & -\frac{i}{24 \pi} \iint A(\mathbf{x})(\mathbf{x}-\mathbf{y})^{2} A(\mathbf{y}) \chi^{\dagger}(\mathbf{y})(1+\beta) \chi(\mathbf{x}) d^{3} y d^{3} x \\
& -\frac{1}{12 \pi} \iint A(\mathbf{x}) \sum_{j=1}^{3} A(\mathbf{y}) \chi^{\dagger}(\mathbf{y}) \alpha_{j}\left(x_{j}-y_{j}\right) \chi(\mathbf{x}) d^{3} y d^{3} x \\
& +\frac{i}{8 \pi m} \iint A(\mathbf{x}) A(\mathbf{y}) \chi^{\dagger}(\mathbf{y}) \chi(\mathbf{x}) d^{3} y d^{3} x \\
=: & s_{1}+s_{2}+s_{3} .
\end{aligned}
$$

For $s_{1}$ we can write

$$
\begin{gathered}
s_{1}=-\frac{i}{24 \pi} \iint A(\mathbf{x})\left(\mathbf{x}^{2}+\mathbf{y}^{2}\right) A(\mathbf{y}) \chi^{\dagger}(\mathbf{y})(1+\beta) \chi(\mathbf{x}) d^{3} y d^{3} x \\
+\frac{i}{12 \pi} \iint A(\mathbf{x}) \mathbf{x} \cdot \mathbf{y} A(\mathbf{y}) \chi^{\dagger}(\mathbf{y})(1+\beta) \chi(\mathbf{x}) d^{3} y d^{3} x .
\end{gathered}
$$

Using symmetry in exchanging $\mathbf{x}$ with $\mathbf{y}$ on the first term it becomes

$$
\begin{aligned}
& -\frac{i}{12 \pi} \iint A(\mathbf{x}) \mathbf{x}^{2} A(\mathbf{y}) \chi^{\dagger}(\mathbf{y})(1+\beta) \chi(\mathbf{x}) d^{3} y d^{3} x \\
= & -\frac{i}{12 \pi} \int A(\mathbf{x}) \mathbf{x}^{2} \chi^{\dagger}(\mathbf{x}) \int(1+\beta) A(\mathbf{y}) \chi(\mathbf{y}) d^{3} y d^{3} x=0
\end{aligned}
$$


by (15). Thus

$$
s_{1}=\frac{i}{12 \pi} \int A(\mathbf{x}) \chi^{\dagger}(\mathbf{x}) \mathbf{x} d^{3} x(1+\beta) \cdot \int \mathbf{y} A(\mathbf{y}) \chi(\mathbf{y}) d^{3} y .
$$

Setting

$$
\xi:=(12 \pi)^{-1 / 2} \int A(\mathbf{x}) \chi(\mathbf{x}) \mathbf{x} d^{3} x
$$

we obtain

$$
s_{1}=i\langle\xi(1-\beta), \xi\rangle .
$$

Since $\beta$ is self adjoint it follows that $\langle\xi(1-\beta), \xi\rangle \in \mathbb{R}$, since $\|\beta\|=1$ it follows that $\langle\xi(1-\beta), \xi\rangle \geq 0$ hence there exists a $C_{2} \in \mathbb{R}_{0}^{+}$such that

$$
s_{1}=i C_{2} .
$$

Due to symmetry in exchanging $\mathbf{x}$ with $\mathbf{y}$ we have that

$$
s_{2}=-s_{2}=0 .
$$

For $s_{3}$ we can write

$$
s_{3}=\frac{i}{8 \pi}\left|\int A(\mathbf{x}) \chi(\mathbf{x}) d^{3} x\right|^{2},
$$

it follows that there exists a $C_{3} \geq 0$ with

$$
s_{3}=i C_{3} .
$$

This (96) and (97) in (92) yield that there exists a $C_{1} \geq 0$ such that

$$
s(\chi, \chi)=i C_{1} .
$$

Since $A$ was defined to satisfy either (16) or (17) it follows taking note of (94) and (95) as well as (98) that $C_{2}$ or $C_{3}>0$, hence $C_{1}=C_{2}+C_{3}>0$, i.e. $s(\chi, \chi) \neq 0$.

\section{Proof of part (d) of Lemma 6.5}

Similar as above we have using Taylors formula that

$$
\left(T_{E_{k}}^{A}-1\right) \Phi=\left(T_{1}^{A}-1\right) \Phi+k\left[\partial_{k}\left(T_{E_{k}}^{A}\right) \Phi\right]_{k=0}+\mathcal{O}\left(k^{2}\right) .
$$

Since $\Phi \in \mathcal{N}$

$$
\left(T_{1}^{A}-1\right) \Phi=0 .
$$

It follows that

$$
\left(T_{E_{k}}^{A}-1\right) \Phi=k\left[\partial_{k}\left(T_{E_{k}}^{A}-1\right) \Phi\right]_{k=0}+\mathcal{O}\left(k^{2}\right)\|\Phi\|_{\infty}
$$

and

$$
\left\|\left(T_{E_{k}}^{A}-1\right) \Phi\right\|_{\infty} \leq k\left\|\left[\partial_{k}\left(T_{E_{k}}^{A}-1\right) \Phi\right]_{k=0}\right\|_{\infty}+\mathcal{O}\left(k^{2}\right)\|\Phi\|_{\infty} .
$$

With (85) and (8) we have that by virtue of (15)

$$
\left[\partial_{k}\left(T_{E_{k}}^{A}-1\right)\right]_{k=0} \Phi=\frac{-i}{4 \pi} \int(1+\beta) A(\mathbf{y}) \Phi(\mathbf{y}) d^{3} y .
$$

Using (15) it follows that

$$
\left[\partial_{k}\left(T_{E_{k}}^{A}-1\right) \Phi\right]_{k=0}=\frac{i \lambda(\Phi)}{4 \pi} .
$$


With (101) part (d) follows.

Proof of (e) Using (28) and part (d) of the Lemma yields

$$
\begin{aligned}
\left|\left\langle\left(T_{E_{k}}^{A+B}-T_{1}^{A+B}\right) h, A, \Phi\right\rangle\right| & =\left|\left\langle h, A+B,\left(T_{E_{k}}^{A}-T_{1}^{A}\right) \Phi\right\rangle\right| \\
& \leq\|(A+B) h\|_{1}\left\|\left(T_{E_{k}}^{A}-T_{1}^{A}\right) \Phi\right\|_{\infty} \\
& \leq\|h\|_{\infty}\|A+B\|_{1} C\left(\bar{\lambda} k+k^{2}\right) .
\end{aligned}
$$

Using the triangle inequality part (e) of the Lemma follows.

Proof of (f) Using (28) we have

$$
\begin{aligned}
\left\langle\Phi, A,\left(1-T_{E_{k}}^{A+B}\right) m^{\perp}\right\rangle & =\left\langle\Phi, A,\left(1-T_{1}^{A+B}\right) m^{\perp}\right\rangle+\left\langle\Phi, A,\left(T_{1}^{A+B}-T_{E_{k}}^{A+B}\right) m^{\perp}\right\rangle \\
& =\left\langle\left(1-T_{1}^{A}\right) \Phi, A+B, m^{\perp}\right\rangle+\left\langle\Phi, A,\left(T_{1}^{A+B}-T_{E_{k}}^{A+B}\right) m^{\perp}\right\rangle \\
& =\left\langle\Phi, A,\left(T_{1}^{A+B}-T_{E_{k}}^{A+B}\right) m^{\perp}\right\rangle .
\end{aligned}
$$

In view of part (e) we get part (f) of the Lemma.

Proof of (g) Using the triangle inequality and linearity of $P_{\mathcal{M}}^{\perp}$ and $T_{E_{k}}^{A+B}$ and (28) we have that

$$
\begin{aligned}
\left\|P_{\mathcal{M}}^{\perp}\left(1-T_{E_{k}}^{A+B}\right) m^{\perp}\right\|_{\infty} \geq & \left\|\left(1-T_{E_{k}}^{A+B}\right) m^{\perp}\right\|_{\infty}-\left\|P_{\mathcal{M}}^{\|}\left(1-T_{E_{k}}^{A+B}\right) m^{\perp}\right\|_{\infty} \\
\geq & \left\|\left(1-T_{E_{k}}^{A}\right) m^{\perp}\right\|_{\infty}-\left\|T_{E_{k}}^{B} m^{\perp}\right\|_{\infty} \\
& -\left\|P_{\mathcal{M}}^{\|}\left(1-T_{E_{k}}^{A}\right) m^{\perp}\right\|_{\infty} \\
=: & S_{1}-S_{2}-S_{3} .
\end{aligned}
$$

Using part (a) of the Lemma we have that

$$
S_{1} \geq C
$$

For $S_{2}$ we have

$$
\begin{aligned}
S_{2}= & \left\|T_{E_{k}}^{B} m^{\perp}\right\|_{\infty}=\left\|\int G_{E_{k}}^{+}(\mathbf{x}-\mathbf{y}) B(\mathbf{y}) m^{\perp}(\mathbf{y}) d^{3} y\right\|_{\infty} \\
\leq & \left\|\int_{|\mathbf{x}-\mathbf{y}|<1} G_{E_{k}}^{+}(\mathbf{x}-\mathbf{y}) B(\mathbf{y}) m^{\perp}(\mathbf{y}) d^{3} y\right\|_{\infty} \\
& +\left\|\int_{|\mathbf{x}-\mathbf{y}|>1} G_{E_{k}}^{+}(\mathbf{x}-\mathbf{y}) B(\mathbf{y}) m^{\perp}(\mathbf{y}) d^{3} y\right\|_{\infty} .
\end{aligned}
$$

Since $G^{+}(\mathbf{x})$ is integrable for all $k<k_{0}$ and bounded uniform in $k<k_{0}$ and $x>1$ it follows that there exists a constant $C$ such that

$$
S_{2} \leq C\|B\|_{\infty}+C\|B\|_{1} .
$$

For $S_{3}$ we use part (f) of the Lemma. Choose $\Phi$ in part (f) such that $A \Phi$ is parallel to $P_{\mathcal{M}}^{\|}\left(1-T_{E_{k}}^{A}\right) m^{\perp}$ and normalized. It follows that

$$
\left|\left\langle\Phi, A,\left(1-T_{E_{k}}^{A+B}\right) m^{\perp}\right\rangle\right|=\left|\left\langle\Phi, A, P_{\mathcal{M}}^{\|}\left(1-T_{E_{k}}^{A+B}\right) m^{\perp}\right\rangle+\left\langle\Phi, A, P_{\mathcal{M}}^{\perp}\left(1-T_{E_{k}}^{A+B}\right) m^{\perp}\right\rangle\right|
$$

Using the definition of $P_{\mathcal{M}}^{\perp}$ the second summand is zero, hence (remember that $\Phi$ was defined such that $A \Phi$ is parallel to $\left.P_{\mathcal{M}}^{\|}\left(1-T_{E_{k}}^{A+B}\right) m^{\perp}\right)$

$$
\left|\left\langle\Phi, A,\left(1-T_{E_{k}}^{A+B}\right) m^{\perp}\right\rangle\right|=\left|\left\langle\Phi, A, P_{\mathcal{M}}^{\|}\left(1-T_{E_{k}}^{A+B}\right) m^{\perp}\right\rangle\right|=\left\|P_{\mathcal{M}}^{\|}\left(1-T_{E_{k}}^{A+B}\right) m^{\perp}\right\|=S_{3} .
$$

Using the equivalence of all norms on the finite dimensional vector-space $\mathcal{M}^{\|}$and part (f) of the Lemma it follows that there exists a $C>0$ such that $S_{3}<C\left(\|A\|_{1}+\|B\|_{1}\right)\left(\bar{\lambda} k+k^{2}\right)$. With (102), (103) and (104) part (g) of the Lemma follows. 
Proof of part (h) Since $\Phi \in \mathcal{N}$, i.e. $\Phi=T_{1}^{A} \Phi$ it follows with part (c) of the Lemma that there exists a $C>0$ such that

$$
\begin{aligned}
\left\|P_{\mathcal{M}}^{\perp}\left(1-T_{E_{k}}^{A+B}\right) \Phi\right\|_{\infty} & =\left\|P_{\mathcal{M}}^{\perp}\left(T_{1}^{A}-T_{E_{k}}^{A+B}\right) \Phi\right\|_{\infty} \\
& \leq C\left\|\left(T_{1}^{A}-T_{E_{k}}^{A+B}\right) \Phi\right\|_{\infty} \\
& \leq C\left\|\left(T_{1}^{A}-T_{E_{k}}^{A}\right) \Phi\right\|_{\infty}+C\left\|\left(T_{E_{k}}^{A}-T_{E_{k}}^{A+B}\right) \Phi\right\|_{\infty} \\
& =:
\end{aligned}
$$

For $S_{1}$ we have using part (d) of the Lemma that there exists a $C>0$ such that

$$
S_{1} \leq C\left(\bar{\lambda} k+k^{2}\right) .
$$

$S_{2}$ can be estimated similarly as $S_{2}$ above. We have

$$
\begin{aligned}
S_{2}= & C\left\|\left(T_{E_{k}}^{A}-T_{E_{k}}^{A+B}\right) \Phi\right\|_{\infty}=C\left\|T_{E_{k}}^{B} \Phi\right\|_{\infty} \\
= & C\left\|\int G_{E_{k}}^{+}(\mathbf{x}-\mathbf{y}) B(\mathbf{y}) \Phi(\mathbf{y}) d^{3} y\right\|_{\infty} \\
\leq & C\left\|\int_{|\mathbf{x}-\mathbf{y}|<1} G_{E_{k}}^{+}(\mathbf{x}-\mathbf{y}) B(\mathbf{y}) \Phi(\mathbf{y}) d^{3} y\right\|_{\infty} \\
& +C\left\|\int_{|\mathbf{x}-\mathbf{y}|>1} G_{E_{k}}^{+}(\mathbf{x}-\mathbf{y}) B(\mathbf{y}) \Phi(\mathbf{y}) d^{3} y\right\|_{\infty} .
\end{aligned}
$$

Since $G^{+}(\mathbf{x})$ is integrable for all $k<k_{0}$ and bounded uniform in $k<k_{0}$ and $x>1$ it follows that there exists a constant $C$ such that

$$
S_{2} \leq C\|B\|_{\infty}+C\|B\|_{1} .
$$

With (105) and (106) part (h) of the Lemma follows.

Proof of (j) Using that $\Psi \in \mathcal{N}$, i.e. $\Psi=T_{1}^{A} \Psi$ and linearity of $T_{E_{k}}^{A}$ in $A$ we get

$$
\begin{aligned}
\left\langle\Phi, A,\left(1-T_{E_{k}}^{A+B}\right) \Psi\right\rangle & =\left\langle\Phi, A,\left(T_{1}^{A}-T_{E_{k}}^{A+B}\right) \Psi\right\rangle \\
& =\left\langle\Phi, A,\left(T_{1}^{A+B}-T_{E_{k}}^{A+B}\right) \Psi\right\rangle+\left\langle\Phi, A,\left(T_{1}^{A}-T_{1}^{A+B}\right) \Psi\right\rangle \\
& =\left\langle\Phi, A,\left(T_{1}^{A}-T_{E_{k}}^{A}\right) \Psi\right\rangle+\left\langle\Phi, A,\left(T_{1}^{B}-T_{E_{k}}^{B}\right) \Psi\right\rangle-\left\langle\Phi, A, T_{1}^{B} \Psi\right\rangle .
\end{aligned}
$$

Note, that due to (28)

$$
\left\langle\Phi, A, T_{1}^{B} \Psi\right\rangle=\left\langle\Phi, B, T_{1}^{A} \Psi\right\rangle=\langle\Phi, B, \Psi\rangle .
$$

Using this and (78) on the first, (79) on the second summand in (107) (remember, that we need results for fixed $A$ and rather general $B$, hence the $\left\|(1-x)^{2} A\right\|$ dependence is in the constants) yields part (j) of the Lemma.

\section{References}

[1] Beck, F., Steinwedel, H. and Süssmann, G.: Z.Phys 171, 189-198 (1963)

[2] Daumer, M., Dürr, D., Goldstein, S. and Zanghì, N.: On the flux-across-surfaces theorem, Lett. Math. Phys. 38, no. 1, 103-116 (1996).

[3] Dell'Antonio, G. F. and Panati, G.: Zero-energy resonances and the flux -across-surfaces theorem, math-ph/0110034, 2001.

[4] Duerr, D., Moser, T. and Pickl, P.: The Flux-Across-Surfaces Theorem Under Conditions on the Scattering State, J. Phys. A: Math. Gen. 39 163-183 (2006).

[5] Dürr, D. and Pickl, P.: Flux-Across-Surfaces Theorem for a Dirac-Particle, J. Math. Phys. 44, 423-465 (2003). 
[6] Gershtein, S. and Zeldovich, Y.: Sov. Phys. JETP 30, 358- (1970)

[7] Greiner, W., Müller, B., Rafelski, J.: Quantum Electrodynamics of Strong Fields, Springer Verlag, Berlin (1985).

[8] Ikebe, T.: Eigenfunction Expansions Assoziated with the Schrödinger Operators and their Application to Scattering Theory, Arch. Rat. Mech. Anal. 5, 1-34 (1960).

[9] Jensen, A., Kato, T.: Spectral Properties of Schrödinger Operators and Time-Decay of the Wave Functions, Duke Math. J. 46 no. 3, 583-611 (1979).

[10] Jensen, A., Nenciu, G.: On The Fermi Golden Rule: Degenerate Eigenvalues, Commun. Math. Phys. 261, 693-727 (2006).

[11] Klaus, M.: On Coupling Constant Thresholds and Related Eigenvalue Properties of Dirac Operators, J. Reine Angew.Math. 362 197-212 (1985).

[12] Nenciu, G.: On the Adiabatic Limit for Dirac Particles in External Fields, Commun. Math. Phys. 76, 117-128 (1980).

[13] Nenciu, G.: Existence of Spontaneous Pair Creation in the External Field Approximation of Q.E.D., Commun. Math. Phys. 109, 303-312 (1987).

[14] Pickl, P.: Existence of Spontaneous Pair Creation, Dissertation (2005).

[15] Pickl P. and Dürr D.: Adiabatic Pair Creation in Heavy Ion and Laser Fields, arXiv:hep-th/0609200v2

[16] Pickl, P. and Dürr, D.: Adiabatic Pair Creation, in preparation.

[17] Povzner, A.: On the Expansion of Arbitrary Functions in Terms of Eigenfunctions of the Operator $-\Delta u+c u$, Math. Sbornik 32, 109-156 (1953).

[18] Prodan E.: Spontaneous transitions in quantum mechanics, J. Phys. A: Math. Gen., 32 4877-4881 (1999).

[19] Rein, D.: Über den Grundzustand überschwerer Atome, Z. Phys 221, 423-430 (1969)

[20] Riesz, F. and Nagy, B.: Functional Analysis, New York: F. Ungar. Publ. Co. (1955).

[21] Rodnianski, I., Schlag, W.: Time Decay for Solutions of Schrödinger Equations with Rough and Time-Dependent Potentials, Inventiones Mathematicae 155 451-513 (2004).

[22] Saito, Y. and Umeda, T.: The Zero Modes and Zero Resonances of Massless Dirac Operators, Hokkaido Mathematical Journal, to appear.

[23] Simon, B.: On the absorption of eigenvalues by continuous spectrum in regular perturbation problems, J. Funct. Anal. 25, 338344 (1977)

[24] Teufel, S.: Adiabatic Perturbation Theory in Quantum Dynamics, Springer Verlag, Berlin (2000).

[25] Teufel, S., Dürr, D. and Münch-Berndl, K.: The flux-across-surfaces theorem for short range potentials and wave functions without energy cutoffs, J. Math. Phys. 40 1901-1922 (1999).

[26] Thaller, B.: The Dirac Equation, Springer Verlag, Berlin (1992).

[27] Yamada, O.: Eigenfunction Expansions and Scattering Theory for Dirac Operators, Publ. RIMS. Kyoto Univ., 11, 651-689 (1976) 